\title{
Finite atomistic lattices that can be represented as lattices of quasivarieties
}

by

\author{
K. V. A d a r i cheva (Novosibirsk), W. D z iobiak (Toruń) \\ and V. A. Gorbunov (Novosibirsk)
}

\begin{abstract}
We prove that a finite atomistic lattice can be represented as a lattice of quasivarieties if and only if it is isomorphic to the lattice of all subsemilattices of a finite semilattice. This settles a conjecture that appeared in the context of [11].
\end{abstract}

Introduction. A quasivariety is any universal Horn class of algebraic systems that contains a trivial algebraic system, or equivalently, any class of algebraic systems that is closed under isomorphic images, subsystems, direct products (including direct products of empty families), and ultraproducts. The set of all quasivarieties contained in a given quasivariety $\mathbf{K}$ forms, with respect to inclusion, a lattice denoted by $L_{q}(\mathbf{K})$. In [16], A. I. Mal'cev asked which lattices can be represented up to isomorphism as lattices of the form $L_{q}(\mathbf{K})$, where $\mathbf{K}$ ranges over all quasivarieties. The question has been named in the literature Mal'cev problem for Q-lattices, where a Q-lattice is a lattice isomorphic to any lattice of the form $L_{q}(\mathbf{K})$. So far a complete solution of Mal'cev problem is only known within the class of Boolean lattices ([11]), the class of lattices of convex subsets of partially ordered sets ([2]) and the class of finite distributive lattices ([20]).

In [11], it was shown that every lattice $S_{p}(A)$ of algebraic subsets of an algebraic lattice $A$ ordered by inclusion is a Q-lattice, where by an algebraic subset of $A$ is meant any subset of $A$ that is closed under arbitrary meets and joins of arbitrary chains formed in $A$. Notice that $S_{p}(A)$ is always an atomistic lattice, i.e. every non-zero element of $S_{p}(A)$ is the join of the atoms under it. These and other properties of $S_{p}(A)$ lead in [11] to the question

1991 Mathematics Subject Classification: Primary 06B15, 08C15; Secondary 03C99, 08A99.

Key words and phrases: atomistic lattice, quasivariety, Mal'cev problem, equa-closure operator, semilattice. 
whether every atomistic Q-lattice is isomorphic to $S_{p}(A)$ for some algebraic lattice $A$. Next, it was conjectured that the question should have an affirmative answer at least within the class of finite lattices. The conjecture in a slightly modified but equivalent form postulates that every finite atomistic Q-lattice is isomorphic to a lattice of the form $S u b(P)$, where $P$ is a finite semilattice and $\operatorname{Sub}(P)$ is the lattice of all subsemilattices of $P$ with empty set as zero. The main aim of this paper is to prove that the conjecture is true.

In [1], certain pure lattice-theoretical necessary and sufficient conditions were given for a finite atomistic lattice to be isomorphic to $S u b(P)$; we recall them in Section 2. In this paper we show that they are satisfied by every finite atomistic Q-lattice. We show first in Section 1 that every Q-lattice is biatomic and has a certain map, called an equa-closure operator, defined on it (for the definitions, see Section 1). Next, we show in Sections 3, 4 and 5 that every finite atomistic lattice that is biatomic and admits an equa-closure operator satisfies the conditions given in [1]. As a result we obtain the following

Theorem. For a finite atomistic lattice $L$ the following conditions are equivalent:

(i) $L$ is a Q-lattice;

(ii) $L$ is biatomic and admits an equa-closure operator;

(iii) $L$ is isomorphic to $S u b(P)$ for some finite semilattice $P$;

(iv) $L$ is isomorphic to $L_{q}(\mathbf{K})$ for some quasivariety $\mathbf{K}$ of rings.

We want to mention that the class of all lattices of the form $S u b(P)$ generates the variety of all lattices (see Freese and Nation [8]).

1. Biatomicity and equa-closure operator. A lattice order of any lattice occurring in this paper will be denoted by $\leq$, and the lattice meet and join of any its two elements $a$ and $b$ by $a b$ and $a+b$, respectively. The smallest and greatest elements of a lattice $L$ (if they exist) will be denoted by 0 and 1 , respectively. If $a, b$ are atoms of $L$, then we shall write $a \sim b$ whenever either $a=b$, or $a \neq b$ and the interval $[0, a+b]$ in $L$ consists of $0, a, b$ and $a+b$. A lattice $L$ is said to be atomic if it has 0 and for each $b$ in $L$ different from 0 there exists an atom $a$ of $L$ with $a \leq b$. $L$ is said to be atomistic (see [13] or [19]) if it is atomic and every non-zero element of $L$ is the join of the atoms under it. A lattice $L$ is said to be biatomic (see Bennett [4], also Birkhoff and Bennett [5]) if it is atomic and, for each atom $a$ of $L$ and all $b, c$ in $L, a \leq b+c$ implies $a \leq b^{\prime}+c^{\prime}$ for some atoms $b^{\prime}, c^{\prime}$ of $L$ with $b^{\prime} \leq b$ and $c^{\prime} \leq c$.

The least quasivariety containing a class $K$ of algebraic systems will be denoted by $Q(K)$, and instead of $Q(\{A\})$ we shall write $Q(A)$. A re- 
sult of Mal'cev [17] (see also Grätzer and Lakser [14]) states that $Q(K)=$ $\operatorname{ISPP}_{\mathrm{U}}(K)$, where $\mathrm{I}, \mathrm{S}, \mathrm{P}$ and $\mathrm{P}_{\mathrm{U}}$ denote the operators of forming isomorphic copies, subsystems, direct products (including direct products of empty families), and ultraproducts, respectively. The operator of forming homomorphic images is denoted by $\mathrm{H}$.

We now proceed to show that every Q-lattice is biatomic. First, however, we want to mention that so far only few nontrivial properties of Q-lattices are known to be expressible in the first-order lattice language. The first such property, already noticed by Mal'cev [17], is the atomicity of Q-lattices. The second, observed in [10], is the join-semidistributivity. The third is the property saying that the lattice join of a finite set of $n$ atoms contains at most $2^{n}-1$ atoms below (see [7]). Thus biatomicity is another first-order property that is shared by every Q-lattice. This property together with the existence on a Q-lattice of an equa-closure operator defined below will play an essential role in our considerations.

Proposition 1.1. Every Q-lattice is biatomic.

Proof. Let $L$ be a Q-lattice. Then $L \cong L_{q}(\mathbf{K})$ for some quasivariety $\mathbf{K}$. We show that $L_{q}(\mathbf{K})$ is biatomic. Let $\mathbf{A}, \mathbf{B}$ and $\mathbf{C}$ be elements of $L_{q}(\mathbf{K})$ with $\mathbf{A}$ being an atom and $\mathbf{A} \leq \mathbf{B}+\mathbf{C}$. Notice that it suffices only to show that $\mathbf{A} \leq \mathbf{D}+\mathbf{C}$ for some atom $\mathbf{D}$ of $L_{q}(\mathbf{K})$ with $\mathbf{D} \leq \mathbf{B}$. Let $A$ be a fixed nontrivial algebraic system of $\mathbf{A}$. Since $\mathbf{A}$ is an atom, we have $\mathbf{A}=Q(A)$. So, as $\mathbf{A} \leq \mathbf{B}+\mathbf{C}$ and $\mathbf{B}+\mathbf{C}=\operatorname{ISP}(\mathbf{B} \cup \mathbf{C})$, it follows that there exist congruence relations $\Theta_{B}$ and $\Theta_{C}$ on $A$ with $A / \Theta_{B} \in \mathbf{B}, A / \Theta_{C} \in \mathbf{C}$ and $\Theta_{B} \wedge \Theta_{C}=i d_{A}$ (see [12]). We may assume that $A / \Theta_{B}$ is non-trivial since otherwise $A \in \mathbf{C}$ and hence $\mathbf{A} \leq \mathbf{C}$ from which the biatomicity of $L_{q}(\mathbf{K})$ immediately follows. This assumption gives that $Q\left(A / \Theta_{B}\right) \neq O_{\mathbf{K}}$. So, as $L_{q}(\mathbf{K})$ is atomic, there exists an atom $\mathbf{D}$ of $L_{q}(\mathbf{K})$ with $\mathbf{D} \leq Q\left(A / \Theta_{B}\right)$. Pick a non-trivial algebraic system $D$ from $\mathbf{D}$. As $D \in Q\left(A / \Theta_{B}\right), D$ is isomorphic to a subdirect product of some family of non-trivial algebraic systems belonging to $\operatorname{SP}_{\mathrm{U}}\left(A / \Theta_{B}\right)$. In particular, there exists a homomorphism of $D$ onto a non-trivial system, say $D^{\prime}$, which is a subsystem of some ultrapower, say, $\prod_{\mathrm{U}}\left(A / \Theta_{B}\right)^{I}$, of $A / \Theta_{B}$. Define $\varphi_{0}: \prod_{\mathrm{U}} A^{I} \rightarrow \prod_{\mathrm{U}}\left(A / \Theta_{B}\right)^{I}$ by

$$
\varphi_{0}\left(\left[\left\langle a_{i}: i \in I\right\rangle\right] \Theta_{\mathrm{U}}\right)=\left[\left\langle\left[a_{i}\right] \Theta_{B}: i \in I\right\rangle\right] \Theta_{\mathrm{U}} .
$$

Similarly, define $\varphi_{1}: \prod_{\mathrm{U}} A^{I} \rightarrow \prod_{\mathrm{U}}\left(A / \Theta_{C}\right)^{I}$ by

$$
\varphi_{1}\left(\left[\left\langle a_{i}: i \in I\right\rangle\right] \Theta_{\mathrm{U}}\right)=\left[\left\langle\left[a_{i}\right] \Theta_{C}: i \in I\right\rangle\right] \Theta_{\mathrm{U}} .
$$

Obviously, both maps are homomorphisms and $\operatorname{Ker} \varphi_{0} \wedge \operatorname{Ker} \varphi_{1}=i d_{\Pi_{\mathrm{U}} A^{I}}$ because $\Theta_{B} \wedge \Theta_{C}=i d_{A}$. Hence the map $\varphi(x)=\left(\varphi_{0}(x), \varphi_{1}(x)\right)$ establishes an embedding of $\prod_{\mathrm{U}} A^{I}$ in $\prod_{\mathrm{U}}\left(A / \Theta_{B}\right)^{I} \times \prod_{\mathrm{U}}\left(A / \Theta_{C}\right)^{I}$. Recall that $D^{\prime}$ is a subsystem of $\prod_{\mathrm{U}}\left(A / \Theta_{B}\right)^{I}$. Let $D^{\prime \prime}$ be a subsystem of $\prod_{\mathrm{U}} A^{I}$ that is the pre-image of $D^{\prime}$ under $\varphi_{0}$, and let $C$ be the image of $D^{\prime \prime}$ by $\varphi_{1}$. Then the 
map $\varphi$ restricted to $D^{\prime \prime}$ embeds $D^{\prime \prime}$ in $D^{\prime} \times C$. So, as $D^{\prime}$ is a homomorphic image of $D$, we obtain $D^{\prime \prime} \in \operatorname{HSP}(\{D, C\})$. But $D^{\prime \prime} \in Q(A)$ and hence $Q\left(D^{\prime \prime}\right)=Q(A)$ since $D^{\prime \prime}$ is non-trivial and $Q(A)$ is an atom in $L_{q}(\mathbf{K})$. Therefore, $A \in \operatorname{HSP}(\{D, C\})$. Evidently, $D \in \operatorname{HSP}(A)$ and $C \in \operatorname{HSP}(A)$. Thus $\operatorname{HSP}(A)=\operatorname{HSP}(\{D, C\})$ which, by $\mathbf{A}=Q(A)$ and $\mathbf{D}=Q(D)$, implies $F \in \mathbf{D}+Q(C)$ where $F$ is a free algebraic system of $\mathbf{A}$ with $\omega$ free generators. So, as $\mathbf{A}$ is an atom, $\mathbf{A}=Q(F)$, and, as $Q(C) \leq \mathbf{C}$, we obtain $\mathbf{A} \leq \mathbf{D}+\mathbf{C}$, proving that $L_{q}(\mathbf{K})$ is biatomic and so is $L$.

Given a quasivariety $\mathbf{K}$. Define a map $\mathbf{h}: L_{q}(\mathbf{K}) \rightarrow L_{q}(\mathbf{K})$ by $\mathbf{h}(\mathbf{M})=$ $\mathrm{H}(\mathbf{M}) \cap \mathbf{K}$. It is easy to see that the map has all properties of an abstract closure operator defined on a lattice. The map has, however, its own characteristic properties, independently of what quasivariety is taken as $\mathbf{K}$. It turned out (see [2] and [7]) that discovering the characteristic properties of $\mathbf{h}$ is very helpful for recognizing the inner structure of the lattice $L_{q}(\mathbf{K})$, or more generally, of any Q-lattice. In [2], an approach is proposed to look at $\mathbf{h}$ as an abstract operator acting on a lattice and to isolate its characteristic properties in the form of axioms. Seven such axioms were postulated in [2]. We adjoin to them a new one and show that every finite atomistic and biatomic lattice on which it is possible to define an operator satisfying all those eight axioms meets the conditions given in [1] for a finite atomistic lattice to be isomorphic to $S u b(P)$. This will occupy most of the paper.

Let $L$ be a complete lattice. A function $h: L \rightarrow L$ is said to be an abstract closure operator if, for $a, b \in L$,

(h1) $a \leq h(a)$

(h2) $\quad h(h(a))=h(a)$;

(h3) $\quad a \leq b$ implies $h(a) \leq h(b)$.

Notice that the set $h(L)$ of $h$-closed elements of $L$ is closed under arbitrary meets formed in $L$ and $h(1)=1$. Hence $h(L)$ has the structure of a complete lattice.

An abstract closure operator $h: L \rightarrow L$ is said to be an equa-closure operator (cf. [2]) if, for $a, b, c \in L$,

$$
\begin{aligned}
& h(0)=0 ; \\
& h(a)=h(b) \text { implies } h(a)=h(a b) ; \\
& h(a)(b+c)=h(a) b+h(a) c ;
\end{aligned}
$$

(h7) Every element of $h(L)$ is the lattice meet formed in $L$ of some family of dually compact elements of $L$.

An example of an abstract closure operator satisfying (h4)-(h7) is the map $\mathbf{h}: L_{q}(\mathbf{K}) \rightarrow L_{q}(\mathbf{K})$ defined above (see [2] for the proof). In the sequel, this map will be called the actual equa-closure operator of $L_{q}(\mathbf{K})$. 
The existence on a complete lattice $L$ of an abstract closure operator satisfying (h1)-(h7) yields that the structure of $L$ cannot be arbitrary. For instance, the join of a finite set of $n$ atoms of $L$ can contain at most $2^{n}-1$ atoms below. This property was proved first for $L$ being a Q-lattice and next extended to arbitrary $L$ admitting a map with (h1)-(h7) (see [7] and $[2]$ ). This seems to justify the abstract approach adopted here (see also [2]).

The new axiom we want to adjoin is the following, where $a, b, c, d$ are arbitrary atoms of $L$ :

$$
a \sim d, d \not \leq h(a), d \leq h(c) \text { and } h(c)=h(a+b) \text { imply } h(c)=h(d+b) .
$$

Thus an equa-closure operator is any abstract closure operator satisfying (h4)-(h8). The axiom (h8) has been isolated from the corresponding property of $\mathbf{h}$ (see Proposition 1.2 below).

We say that a complete lattice $L$ admits an equa-closure operator if there exists a map defined on $L$ that satisfies all axioms (h1)-(h8).

Proposition 1.2. For each quasivariety $\mathbf{K}$, the actual equa-closure operator $\mathbf{h}: L_{q}(\mathbf{K}) \rightarrow L_{q}(\mathbf{K})$ satisfies (h8). In particular, every Q-lattice admits an equa-closure operator.

Proof. Let $\mathbf{A}, \mathbf{B}, \mathbf{C}$ and $\mathbf{D}$ be atoms of $L_{q}(\mathbf{K})$ such that $\mathbf{A} \sim \mathbf{D}$, $\mathbf{D} \not \mathbf{h}(\mathbf{A}), \mathbf{D} \leq \mathbf{h}(\mathbf{C})$ and $\mathbf{h}(\mathbf{C})=\mathbf{h}(\mathbf{A}+\mathbf{B})$. Let $F$ denote the free algebraic system in $\mathbf{C}$ with $\omega$ free generators. As $\mathbf{C}$ is an atom, $\mathbf{C}$ is generated by $F$, that is, $\mathbf{C}=\operatorname{ISPP}_{\mathrm{U}}(F)$. As $\mathbf{h}(\mathbf{C})=\mathbf{h}(\mathbf{A}+\mathbf{B})$, the algebraic system $F$ is also free in $\mathbf{h}(\mathbf{A}+\mathbf{B})$, and, therefore, it belongs to $\mathbf{A}+\mathbf{B}$. Hence $F \in \operatorname{ISP}(\mathbf{A} \cup \mathbf{B})$ since $\mathbf{A}+\mathbf{B}=\operatorname{ISPP}_{\mathrm{U}}(\mathbf{A} \cup \mathbf{B})=\operatorname{ISP}(\mathbf{A} \cup \mathbf{B})$. So there exist congruence relations $\Theta_{A}$ and $\Theta_{B}$ on $F$ such that $F / \Theta_{A} \in \mathbf{A}, F / \Theta_{B} \in \mathbf{B}$ and $\Theta_{A} \wedge \Theta_{B}=i d_{F}$. We may of course assume that $F / \Theta_{A}$ is non-trivial since otherwise $F \in \mathbf{B}$ and then $\mathbf{h}(\mathbf{C})=\mathbf{h}(\mathbf{D}+\mathbf{B})$. As $\mathbf{A}$ is an atom, the assumption implies that $F / \Theta_{A}$ generates $\mathbf{A}$. On the other hand, as $\mathbf{D} \leq \mathbf{h}(\mathbf{C})$ and $\mathbf{D}$ is an atom, there must exist a congruence relation $\Theta_{D}$ on $F$ such that $F / \Theta_{D}$ generates $\mathbf{D}$. We may assume that $\mathbf{A} \not \leq \mathbf{h}(\mathbf{D})$ since otherwise the conclusion is immediate. We claim that the quotient system $F / \Theta_{A} \wedge \Theta_{D}$ generates $\mathbf{A}+\mathbf{D}$. Evidently, $F / \Theta_{A} \wedge \Theta_{D} \in \mathbf{A}+\mathbf{D}$ and, as the lattice $L_{q}(\mathbf{K})$ is atomic and $\mathbf{A} \sim \mathbf{D}$, it follows that either $\mathbf{A}=Q\left(F / \Theta_{A} \wedge\right.$ $\left.\Theta_{D}\right)$, or $\mathbf{D}=Q\left(F / \Theta_{A} \wedge \Theta_{D}\right)$, or $\mathbf{A}+\mathbf{D}=Q\left(F / \Theta_{A} \wedge \Theta_{D}\right)$. Since $F / \Theta_{A}$ generates $\mathbf{A}$ and $F / \Theta_{D}$ generates $\mathbf{D}$, we have $\mathbf{A} \leq \mathbf{h}\left(Q\left(F / \Theta_{A} \wedge \Theta_{D}\right)\right)$ and $\mathbf{D} \leq \mathbf{h}\left(Q\left(F / \Theta_{A} \wedge \Theta_{D}\right)\right)$. So, as $\mathbf{A} \not \leq \mathbf{h}(\mathbf{D})$ and $\mathbf{D} \not \leq \mathbf{h}(\mathbf{A})$, we obtain $\mathbf{A}+\mathbf{D}=Q\left(F / \Theta_{A} \wedge \Theta_{D}\right)$ which proves the claim.

As $\mathbf{A} \not \mathbf{h}(\mathbf{D})$, applying (h6) we obtain $\mathbf{h}(\mathbf{D})(\mathbf{A}+\mathbf{D})=\mathbf{D}$. It follows that $\mathbf{D}$ is generated by an algebraic system, say, $D$, that is subdirectly irreducible in $\mathbf{A}+\mathbf{D}$. By the above claim $D$ is isomorphic to a subsystem, say, $D^{\prime}$, of some ultrapower $\prod_{\mathrm{U}}\left(F / \Theta_{A} \wedge \Theta_{D}\right)^{I}$ of $F / \Theta_{A} \wedge \Theta_{D}$. 
Now, define $\varphi_{0}: \prod_{\mathrm{U}} F^{I} \rightarrow \prod_{\mathrm{U}}\left(F / \Theta_{A} \wedge \Theta_{D}\right)^{I}$ by

$$
\varphi_{0}\left(\left[\left\langle a_{i}: i \in I\right\rangle\right] \Theta_{\mathrm{U}}\right)=\left[\left\langle\left[a_{i}\right] \Theta_{A} \wedge \Theta_{D}: i \in I\right\rangle\right] \Theta_{\mathrm{U}} .
$$

Similarly, define $\varphi_{1}: \prod_{\mathrm{U}} F^{I} \rightarrow \prod_{\mathrm{U}}\left(F / \Theta_{B}\right)^{I}$ by

$$
\varphi_{1}\left(\left[\left\langle a_{i}: i \in I\right\rangle\right] \Theta_{\mathrm{U}}\right)=\left[\left\langle\left[a_{i}\right] \Theta_{B}: i \in I\right\rangle\right] \Theta_{\mathrm{U}} .
$$

Both maps are homomorphisms and, as $\Theta_{A} \wedge \Theta_{D} \wedge \Theta_{B}=i d_{F}$ because $\Theta_{A} \wedge$ $\Theta_{B}=i d_{F}, \operatorname{Ker} \varphi_{0} \wedge \operatorname{Ker} \varphi_{1}=i d_{\Pi_{U} F^{I}}$. Hence the map $\varphi(x)=\left(\varphi_{0}(x), \varphi_{1}(x)\right)$ establishes an embedding of $\prod_{\mathrm{U}} F^{I}$ in $\prod_{\mathrm{U}}\left(F / \Theta_{A} \wedge \Theta_{D}\right)^{I} \times \prod_{\mathrm{U}}\left(F / \Theta_{B}\right)^{I}$. Denote by $D^{\prime \prime}$ a subsystem of $\prod_{U} F^{I}$ that is the pre-image of $D^{\prime}$ under $\varphi_{0}$, and by $B$ the homomorphic image of $D^{\prime \prime}$ by $\varphi_{1}$. Then $\varphi$ restricted to $D^{\prime \prime}$ embeds $D^{\prime \prime}$ in $D^{\prime} \times B$ and $\varphi_{0}\left(D^{\prime \prime}\right)=D^{\prime}$. Hence, as $D^{\prime} \in \mathbf{D}$ and $B \in \mathbf{B}$, it follows that $D^{\prime \prime} \in \mathbf{D}+\mathbf{B}$. Notice that $D^{\prime \prime}$ generates $\mathbf{C}$ because $D^{\prime \prime}$ is non-trivial and $\mathbf{C}$ is an atom. Thus $\mathbf{C} \leq \mathbf{D}+\mathbf{B}$ and, therefore, $\mathbf{h}(\mathbf{C})=\mathbf{h}(\mathbf{D}+\mathbf{B})$ since $\mathbf{D}, \mathbf{B} \leq h(\mathbf{C})$, showing that $h$ satisfies (h8).

The following example shows that (h8) does not follow from (h1)-(h7). Let $P$ be a meet semilattice whose diagram is given in Figure 1 , and let $\varepsilon$ be a binary relation defined on $P$ by $x \varepsilon y$ iff $x=5,6,7,8, y=1,2,3,4$ and $x \leq y$ in $P$. Denote by $\operatorname{Sub}(P, \varepsilon)$ the lattice of all subsemilattices of $P$, including empty set, that are closed under $\varepsilon$, where a subset $X$ of $P$ is closed under $\varepsilon$ if, for all $x, y$ in $P, x \in X$ and $x \varepsilon y$ imply $y \in X$. Define $h: S u b(P, \varepsilon) \rightarrow \operatorname{Sub}(P, \varepsilon)$ by $h(X)=X$ if $9 \notin X$, and $h(X)=P$ otherwise. Obviously, $h$ satisfies (h1)-(h5) and, as $\operatorname{Sub}(P, \varepsilon)$ is finite, $h$ satisfies (h7). To verify (h6) we need to show that $h(X)(Y+Z) \leq h(X) Y+h(X) Z$, where $X, Y, Z \in \operatorname{Sub}(P, \varepsilon)$. If $9 \in X$, this is obvious since in this case $h(X)=P$. So let $9 \notin X$. Then $h(X)=X$ and we need to show that $X(Y+Z) \leq X Y+X Z$. But $\operatorname{Sub}(P, \varepsilon)$ is atomistic with atoms $\{i\}$, where $i=1,2,3,4,9$. So, as $\{i\} \leq$ $Y+Z$, for $i=1,2,3,4$, implies $\{i\} \in Z$, it follows that $X(Y+Z) \leq X Y+X Z$ whenever $9 \notin X$. Notice now that $\{1\} \sim\{2\},\{2\} \not \leq h(\{1\}),\{2\} \leq h(\{9\})$ and $h(\{9\})=h(\{1\}+\{4\})$. Hence, as $h(\{9\}) \neq h(\{2\}+\{4\})$, the map $h$ does not satisfy (h8).

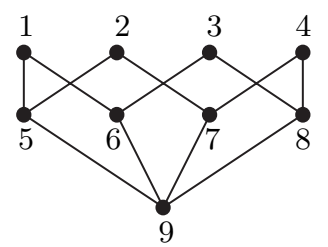

Fig. 1

The lattice $L$ of Figure 2 shows that biatomicity does not follow from the existence on a lattice of an equa-closure operator. Indeed, define $h: L \rightarrow L$ by $h(x)=1$ if $b \leq x$, and $h(x)=x$ otherwise. Then $h$ satisfies (h1)-(h8). 
On the other hand, as $b \leq a+(c+d), b \not \leq a+c$ and $b \not \leq a+d, L$ is not biatomic.

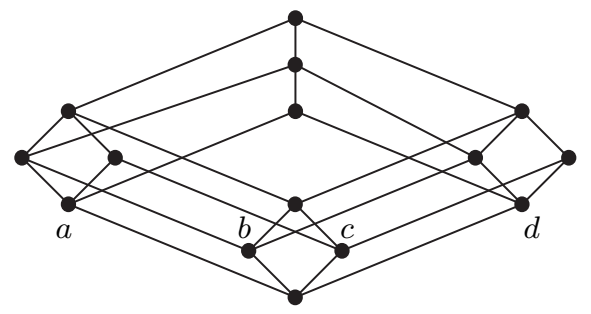

Fig. 2

2. Finite lattices of subsemilattices. We recall in this section the necessary and sufficient conditions given in [1] for a finite atomistic lattice to be isomorphic to $S u b(P)$.

A finite lattice $L$ is said to satisfy $D_{2}$ if, for each pair $a, b$ of atoms of $L$, the interval $[0, a+b]$ in $L$ contains at most 3 atoms of $L$. Recall from Section 1 that $a \sim b$ means that either $a=b$, or $a \neq b$ and the interval $[0, a+b]$ in $L$ consists of $0, a, b$ and $a+b$. If $L$ is atomistic then $a \sim b$ is equivalent to the property that $[0, a+b]$ contains at most 2 atoms of $L$. If $a, b$ and $c$ are atoms of $L$, we write $c \unlhd a+b$ to denote that $c \leq a+b$ and $c \notin\{a, b\}$.

A sequence $a_{0}, a_{1}, \ldots, a_{n}$ of atoms of $L$, where $n \geq 2$, is said to be a cycle if $a_{0}=a_{n}$ and, for each $i<n$, there exists an atom $b_{i}$ of $L$ with $a_{i+1} \unlhd a_{i}+b_{i}$. It is not hard to see that a finite atomistic lattice has no cycles if and only if it is lower bounded in the sense of McKenzie [18] (see also Day [6] and Jónnson and Nation [15]).

Let $a$ and $b$ be atoms of $L$. A sequence $\left(a_{1}, b\right), \ldots,\left(a_{n}, b\right)$ of pairs of atoms of $L$ is said to be a left descent from $(a, b)$ if it satisfies the following conditions:

(i) $a_{1}=a$;

(ii) For each atom $c$ of $L, b \sim c$ implies $a_{n} \sim c$;

(iii) If $2 \leq n$ then, for each $i<n, a_{i+1} \unlhd a_{i}+b_{i}$ for some atom $b_{i}$ of $L$ with $b_{i} \sim b$.

Similarly, we define a right descent from $(a, b)$ as a sequence $\left(b, a_{1}\right), \ldots$ $\ldots,\left(b, a_{n}\right)$ of pairs of atoms of $L$ satisfying (i)-(iii). If $n=1$, the descent will be called trivial. If it is not trivial, we write $\left(a_{1}, b\right){ }^{b_{1}} \nearrow\left(a_{2}, b\right){ }^{b_{2}} \nearrow$ $\ldots{ }^{b_{n-1}} \nearrow\left(a_{n}, b\right)$ if it is left, and $\left(b, a_{1}\right) \searrow^{b_{1}}\left(b, a_{2}\right) \searrow^{b_{2}} \ldots \searrow^{b_{n-1}}\left(b, a_{n}\right)$ if it is right, to emphasize that the descents have been formed with the help of the sequence $b_{1}, \ldots, b_{n}$. 
We say that $L$ has univocally terminating left descents if, for each pair $a, b$ of atoms of $L$ with $a \sim b$ and any two left descents $\left(a_{1}, b\right), \ldots,\left(a_{n}, b\right)$ and $\left(c_{1}, b\right), \ldots,\left(c_{m}, b\right)$ from $(a, b)$, we have $a_{n}=c_{m}$. Notice that the concept can be equivalently expressed in terms of right descents.

A sequence $(a, b), \ldots,(c, d)$ of pairs of atoms of $L$ is said to be a slalom with the origin at $(a, b)$ if it consists of alternating non-trivial left and right descents the last pairs of which are the first pairs of the subsequent descents. The sequences $(\{1\},\{3\})\{4\} /(\{9\},\{3\}) \searrow^{\{2\}}(\{9\},\{9\})$ and $(\{1\},\{3\}) \searrow^{\{2\}}$ $(\{1\},\{9\}){ }^{\{4\}} \nearrow(\{9\},\{9\})$ are examples of slaloms in the lattice $\operatorname{Sub}(P, \varepsilon)$, where $P$ is the semilattice of Figure 1.

A slalom is said to be even (odd) if the number of alternating descents in it is even (odd), and left (right) if its first descent is left (right), and is said to be exact if its last pair has equal components. The above two slaloms in $\operatorname{Sub}(P, \varepsilon)$ are even and exact, the first of them is left while the second is right.

We say that the right and left slaloms in a lattice $L$ have different parities if, for each pair $a, b$ of atoms of $L$ with $a \sim b$, there are no right and left slaloms with the origin at $(a, b)$ that are exact and are both even or both odd.

The following theorem provides pure lattice-theoretical conditions for a finite atomistic lattice to be isomorphic to $S u b(P)$. Actually, the theorem provides conditions for a finite lattice to be isomorphic to $S u b(P)$ because the lattice $\operatorname{Sub}(P)$ is always atomistic.

TheOrem 2.1 ([1]). A finite atomistic lattice $L$ is isomorphic to the lattice $\operatorname{Sub}(P)$ for some finite semilattice $P$ iff it satisfies $D_{2}$, has no cycles, is biatomic, has univocally terminating left descents and the right and left slaloms in L have different parities.

3. A partial semilattice. In this section assuming that $L$ is a lattice admitting an equa-closure operator we define on the set $A(L)$ of atoms of $L$ a partial semilattice operation $\circ$. This operation will be helpful in proving that if, in addition, $L$ is finite then $L$ has univocally terminating left descents, and the right and left slaloms in it have different parities.

Lemma 3.1. Suppose that $L$ is a lattice with an equa-closure operator $h$ and $a, b \in A(L)$. Then the interval $[0, a+b]$ contains at most 3 atoms of $L$. Moreover, if $[0, a+b]$ contains 3 atoms of $L$ then the atoms $a, b$ and $c$ it contains satisfy $a+b \leq h(c), c \not \leq h(a)$ and $c \not \leq h(b)$.

Proof. The first part is obvious since as mentioned earlier the existence of an equa-closure operator on a lattice yields that the join of a finite set of $n$ atoms contains at most $2^{n}-1$ atoms below. To show that $a+b \leq h(c)$ notice that, by (h6), we have $h(c)(a+b)=h(c) a+h(c) b$ and that it suffices 
to prove $h(c) a \neq 0$ and $h(c) b \neq 0$. But if $h(c) a=0$ then $h(c)(a+b)=b$ and, as $c \leq a+b$ and $c \leq h(c)$, it follows that $c=b$, a contradiction. Similarly, $h(c) b \neq 0$. Now, suppose that $c \leq h(a)$. Then, by $a+b \leq h(c)$ just proved, we have $h(a+b)=h(a)$ which, again by $a+b \leq h(c)$ and $c \leq a+b$, implies $h(a)=h(c)$. Hence, by (h1) and (h5), we obtain $a=c$, a contradiction. Thus $c \not \leq h(a)$, and similarly $c \not \leq h(b)$.

Recall from Section 2 that if $a, b$ and $c$ are atoms of a lattice $L$, then $c \unlhd a+b$ means that $c \leq a+b$ and $c \notin\{a, b\}$. We define a ternary relation $r$ on $A(L)$ as follows: $r(a, b, c)$ holds iff either $c \unlhd a+b$, or $c=a$ and $a \unlhd b+d$ for some $d \in A(L)$, or $c=b$ and $b \unlhd a+d$ for some $d \in A(L)$.

Lemma 3.2. Suppose $L$ is a lattice that admits an equa-closure operator and $a, b, c, d \in A(L)$. Then $r(a, b, c)$ and $r(a, b, d)$ imply $c=d$.

P r o of. Let $h$ be an equa-closure operator on $L$ and assume that $r(a, b, c)$ and $r(a, b, d)$ hold. We consider three cases depending on which of the disjuncts defining $r(a, b, c)$ is satisfied.

Cas e 1: $c \unlhd a+b$. As $r(a, b, d)$ holds, one of the disjuncts defining it is satisfied. If it is the first then, as $a+b$ contains exactly 3 atoms below (see Lemma 3.1), we have $c=d$. So, assume that $d=a$ and $a \unlhd b+e$ for some $e \in A(L)$. By Lemma 3.1, we have $b \leq h(a)$ and, as $c \unlhd a+b$, also $h(c)=h(a+b)$. This, by (h1)-(h3), implies $h(c)=h(a)$ which in turn, by (h1) and (h5), gives $c=a$ and hence $c=d$. Assume now that $d=b$ and $b \unlhd a+e$ for some $e \in A(L)$. So, by Lemma 3.1, $a \leq h(b)$ and $h(c)=h(a+b)$ which gives $h(c)=h(b)$ and, therefore, $c=b$ by (h1) and (h5). Thus $c=d$.

Case 2: $c=a$ and $a \unlhd b+e$ for some $e \in A(L)$. If $d \unlhd a+b$ (recall that $r(a, b, d))$ then similarly to Case 1 we obtain $c=d$. If the second disjunct for $r(a, b, d)$ is satisfied then, as $c=a$, we trivially get $c=d$. So assume that $d=b$ and $b \unlhd a+f$ for some $f \in A(L)$. Then, by Lemma 3.1, $a \leq h(b)$ and, as $a \unlhd b+e, b \leq h(a)$. Thus $h(a)=h(b)$ and, therefore, $a=b$ and $c=d$.

Case 3: $c=b$ and $b \unlhd a+e$ for some $e \in A(L)$. If $r(a, b, d)$ is due to the first disjunct then as in Case 1 we obtain $c=d$. If $r(a, b, d)$ is due to the second then as in Case 2 we get $c=d$. In the case of the third disjunct for $r(a, b, d)$, we have $d=b$ and thus $c=d$.

Let $L$ be a lattice that admits an equa-closure operator. Notice that $r(a, a, c)$ never holds in $L$. By Lemma 3.2 we can define a partial binary operation $\circ$ on $A(L)$ as follows:

$$
a \circ b= \begin{cases}a & \text { if } a=b, \\ c & \text { if } r(a, b, c) .\end{cases}
$$

In general, the operation $\circ$ is partial. For the lattice $L$ of Figure 3 define $h: L \rightarrow L$ by $h(x)=1$ if $b \leq x$, and $h(x)=x$ otherwise. Notice that $h$ is an 
equa-closure operator and that the only triples of $r$ here are: $(a, c, b),(c, a, b)$, $(c, d, b),(d, c, b),(a, b, b),(b, a, b),(b, d, b),(d, b, b),(c, b, b)$ and $(b, c, b)$. So $\circ$ is not defined on $(a, d)$ and $(d, a)$.

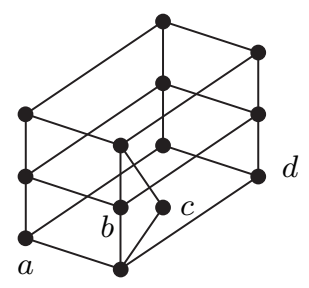

Fig. 3

Writing $a \circ b$ we shall always mean that $\circ$ is defined on $(a, b)$ and sometimes we shall additionally stress this by writing " $a \circ b$ is defined".

Lemma 3.3. Suppose that $L$ is a lattice and $h$ is an equa-closure operator defined on $L$. Then the following conditions hold, where $a, b, c$ are atoms of $L$ :

(i) $h(c)=h(a+b)$ implies $c \leq a+b$;

(ii) $h(a \circ b)=h(a+b)$.

P r o of. (i) Applying (h5) to $h(c)=h(a+b)$ we obtain $h(c)=h(c(a+b))$. As $h(c) \neq 0$ because $c \leq h(c)$ by (h1), the equality implies $c(a+b) \neq 0$. So, as $c$ is an atom, it follows that $c \leq a+b$.

(ii) When $a=b$, the condition is obvious. So assume that $a \neq b$ and that $a \circ b$ is defined. Then either $a \circ b \unlhd a+b$ or $a \circ b \in\{a, b\}$. If $a \circ b \unlhd a+b$ then, by Lemma 3.1, $h(a \circ b)=h(a+b)$. If $a \circ b=a$ then $r(a, b, a)$ and, therefore, $b \leq h(a)$ which implies $h(a)=h(h(a))=h(h(a)+b)=h(a+b)$, and hence $h(a \circ b)=h(a+b)$. If $a \circ b=b$ then $r(a, b, b)$, and the argument is similar.

Proposition 3.4. If $L$ is a lattice that admits an equa-closure operator then $(A(L) ; \circ)$ is a partial semilattice.

Proof. That the operation is idempotent is clear. The (partial) commutativity of $\circ$ follows from Lemma 3.3. For the proof of the (partial) associativity of $\circ$ assume that $a, b, c$ are atoms such that $b \circ c, a \circ(b \circ c)$, $a \circ b,(a \circ b) \circ c$ are defined. Let $h$ be an equa-closure operator on $L$. By Lemma 3.3(ii), we have $h(a \circ(b \circ c))=h(a+b+c)$ and $h((a \circ b) \circ c)=h(a+b+c)$. Hence, applying Lemma 3.3(i) we obtain $a \circ(b \circ c)=(a \circ b) \circ c$.

We call a subset $X$ of $A(L)$ a relative partial subsemilattice of $(A(L) ; \circ)$ if, for all $a, b$ in $L, a \circ b \in X$ whenever $a \circ b$ is defined and $a, b \in X$. It is clear that the set of all relative partial subsemilattices of $(A(L) ; \circ)$ forms a complete lattice with respect to inclusion. 
Proposition 3.5. Suppose $L$ is an algebraic, atomistic and biatomic lattice that admits an equa-closure operator. Then $L$ is isomorphic to the lattice of all relative partial subsemilattices of $(A(L) ; \circ)$.

Proof. Define $\varphi(x)=\{a \in A(L): a \leq x\}$ for $x \in L$. We show that $\varphi$ is an isomorphism between $L$ and the lattice of all relative partial subsemilattices of $(A(L) ; \circ)$. Let $a, b \in \varphi(x)$ and assume that $a \circ b$ is defined. Then $a \circ b \leq a+b$ which implies $a \circ b \in \varphi(x)$ and gives that $\varphi$ is well-defined. That $\varphi$ is one-to-one follows from the atomisticity of $L$. That $\varphi$ preserves meets is obvious. For joins apply the biatomicity of $L$. To see that $\varphi$ is onto use the biatomicity of $L$ and the assumption that $L$ is algebraic. Thus $\varphi$ is an isomorphism.

Having a lattice $L$ with the properties of Proposition 3.5 one can ask whether the partial semilattice operation $\circ$ can be extended to a total semilattice operation $o^{\prime}$ on $A(L)$ so that every relative partial subsemilattice of $(A(L) ; \circ)$ would be a subsemilattice of $\left(A(L) ; \circ^{\prime}\right)$ and vice versa. A positive answer to this question would give us that $L$ is isomorphic to the lattice of all subsemilattices of $\left(A(L) ; \circ^{\prime}\right)$.

If in addition $L$ is finite, we can take for $o^{\prime}$ the semilattice operation defined in the proof of the "if" part of Theorem 2.1, though we have not shown yet that all conditions of Theorem 2.1 are satisfied by $L$. The proof that $L$ satisfies $D_{2}$ and has no cycles is relatively easy and was already given in [1]. Indeed, that $L$ satisfies $D_{2}$ follows from Lemma 3.1. In order to show that $L$ has no cycles suppose on the contrary that $a_{0}, a_{1}, \ldots, a_{n}$, where $n \geq 2$, is a cycle in $L$. Applying Lemma 3.3(ii) we have $a_{i} \leq h\left(a_{i+1}\right)$ for all $i<n$ which, by $a_{0}=a_{n}$, implies $h\left(a_{i}\right)=h\left(a_{n}\right)$ for all $i<n$. Now referring to Lemma 3.3(i) we get $a_{n-1}=a_{n}$, a contradiction since $a_{n} \unlhd a_{n-1}+b_{n-1}$. It is much harder to show that $L$ satisfies the remaining two conditions of Theorem 2.1. This will be done in the next two sections.

4. Univocal termination of left descents. In this section we prove that every finite atomistic lattice $L$ that admits an equa-closure operator has univocally terminating left descents. In proving this we shall refer to the structure of the partial semilattice $(A(L) ; \circ)$ defined in Section 3.

Throughout this section $L$ is a finite atomistic lattice with an equaclosure operator $h$, and $a \sqsubseteq b$, where $a, b \in A(L)$, means that $b \leq h(a)$. Notice that by Lemma 3.3(i) the relation $\sqsubseteq$ is a partial order on $A(L)$.

LEMma 4.1. The following conditions are satisfied for $a, b, c \in A(L)$ :

(i) If $a \circ b$ is defined then so is $a \circ(a \circ b)$ and $a \circ(a \circ b)=a \circ b$;

(ii) If $a \circ b$ is defined and $a \circ b \sqsubseteq c \sqsubseteq a$ then $c \circ b$ is defined and $c \circ b=a \circ b$;

(iii) If $a \circ c$ and $b \circ c$ are defined, $a \circ c=a$ and $b \circ c=b$ then $a \circ b$ is defined. 
Proof. (i) If $a \not z b$ then $a \circ b \unlhd a+b$ and so $r(a, a \circ b, a \circ b)$ which means that $a \circ(a \circ b)$ is defined and $a \circ(a \circ b)=a \circ b$. If $a \sim b$ then, as $a \circ b$ is defined, $a \circ b=a$ or $a \circ b=b$. Assume $a \circ b=a$. Then $a \circ(a \circ b)$ is defined and $a \circ(a \circ b)=a$. If $a \circ b=b$, then $a \circ(a \circ b)$ is defined and $a \circ(a \circ b)=a \circ b$.

(ii) Notice that $h(a \circ b)=h(c+b)$. By Lemma 3.3(i), $a \circ b \leq c+b$. If $a \circ b \unlhd c+b$ then $c \circ b$ is defined and $c \circ b=a \circ b$. Otherwise we have $a \circ b=c$ or $a \circ b=b$. If $a \circ b=c$ then, by the commutativity of $\circ, b \circ a=c$ which, by (i) just proved, implies that $b \circ c$ is defined and $b \circ c=b \circ a$. Thus $c \circ b$ is defined and $c \circ b=a \circ b$. So consider the case when $a \circ b=b$. If $a=b$ then evidently $c \circ b$ is defined and $c \circ b=a \circ b$ because $a=b$ implies $a=c$. So we may assume that $a \neq b$. Then $b \unlhd a+d$ for some $d \in A(L)$. As $a \circ d \sqsubseteq c \sqsubseteq a$ because $b \sqsubseteq c \sqsubseteq a$, applying Lemma 3.3(ii) we obtain $h(a \circ d)=h(c+a \circ d)=h(c+a+d)=h(c+d)$. Hence $a \circ d \leq c+d$ by Lemma 3.3(i). It follows that $a \circ d=c$ or $a \circ d \unlhd c+d$; the case $a \circ d=d$ is excluded since $a \circ d \unlhd a+d$. If $a \circ d=c$ then $a \circ b=c$ which gives the case considered before. If $a \circ d \unlhd c+d$ then $c \circ d$ is defined and $a \circ d=c \circ d$. So, as $b=a \circ d$ (recall that $a \circ b=b$ and $b=a \circ d$ ), we obtain $b=c \circ d$ which, by (i) just proved, yields that $c \circ b$ is defined and $c \circ b=c \circ d=a \circ b$.

(iii) We may assume that $a \neq c$ and $b \neq c$ since otherwise it is easily seen that $a \circ b$ is defined. This implies that $a \unlhd c+d$ and $b \unlhd c+e$ for some atoms $d$, e of $L$. We need to show that $\circ$ is defined on the pair $(a, b)$. Suppose on the contrary that this is not the case.

Claim 1. $(a+e)(a+b)=a$.

Proof. As $\circ$ is not defined on $(a, b)$, the join $a+b$ contains only two atoms under itself, that is, $a$ and $b$. Thus $(a+e)(a+b)=a$ or $=b$ or $=a+b$ since $L$ is atomistic. We show that $(a+e)(a+b) \notin\{b, a+b\}$. Indeed, otherwise $(a+e)(a+b) \in\{b, a+b\}$, and hence $b \leq a+e$. So, as $b$ is an atom, by Lemma 3.1 it follows that $b=a$ or $b=e$ or $b \unlhd a+e$. If $b=a$ or $b \unlhd a+e$ then $\circ$ is defined on $(a, b)$, a contradiction. If $b=e$ then we have a contradiction with $b \unlhd c+e$. Thus $(a+e)(a+b)=a$.

Claim 2. $a(b+d)=0$.

Proof. As $a$ is an atom, supposing the claim does not hold we have $a \leq b+d$. Then, by Lemma 3.1, $a=b$ or $a=d$ or $a \unlhd b+d$. If $a=b$ or $a \unlhd b+d$ then $\circ$ is defined on $(a, b)$, contradicting our assumption. The case $a=d$ also yields a contradiction since $a \unlhd c+d$. Thus $a \not \leq b+d$ which proves that $a(b+d)=0$.

Now, define $X$ to be the set of all $x$ in $L$ such that $h(x)=h(c+d+e)$. Recall that $a=c \circ d$ and $b=c \circ e$. So, applying Lemma 3.3(ii) we have

$$
h(c+d+e)=h(c \circ d+e)=h(a+e),
$$




$$
\begin{aligned}
& h(c+d+e)=h(c \circ d+e)=h(a+e)=h(a+c+e)=h(a+b), \\
& h(c+d+e)=h(c+e+d)=h(c \circ e+d)=h(b+d) .
\end{aligned}
$$

This gives that $a+e, a+b, b+d \in X$. So, by (h5), $(a+e)(a+b)(b+d) \in X$ which together with Claims 1 and 2 shows that $0 \in X$. Hence, by (h4), we obtain $h(c+d+e)=0$ which, by (h1), implies $c=d=e=0$, a contradiction. Thus $\circ$ must be defined on $(a, b)$.

Lemma 4.2. Suppose $a \in A(L)$ and $c_{1}, \ldots, c_{n}, n \geq 2$, is a sequence in $A(L)$ with the following properties:

(i) $a \nsim c_{i}$ for all $i=1, \ldots, n$,

(ii) $c_{i} \not \leq h\left(a+c_{1}+\ldots+c_{i-1}\right)$ for all $i=2, \ldots, n$.

Then each of the expressions $\left(\ldots\left(a \circ c_{1}\right) \circ c_{2} \ldots\right) \circ c_{i}$, where $i=2, \ldots, n$, is defined in $(A(L) ; \circ)$.

P r o of (By induction on $n$ ). For $n=2$, from the property (i) and the definition of $\circ$ it follows that $a \circ c_{1}$ and $a \circ c_{2}$ are defined which, by Lemma 4.1(i) and (iii), implies that so is $\left(a \circ c_{1}\right) \circ\left(a \circ c_{2}\right)$. Applying Lemma 3.3(ii) we have $h\left(\left(a \circ c_{1}\right) \circ\left(a \circ c_{2}\right)\right)=h\left(a \circ c_{1}+c_{2}\right)$ which, by Lemma 3.1, yields $\left(a \circ c_{1}\right) \circ\left(a \circ c_{2}\right) \in\left\{a \circ c_{1}, c_{2}\right\}$ or $\left(a \circ c_{1}\right) \circ\left(a \circ c_{2}\right) \unlhd a \circ c_{1}+c_{2}$. But $c_{2} \leq h\left(\left(a \circ c_{1}\right) \circ\left(a \circ c_{2}\right)\right)$. So, as $c_{2} \not \leq h\left(a+c_{1}\right)$, we have $\left(a \circ c_{1}\right) \circ\left(a \circ c_{2}\right) \neq a \circ c_{1}$. On the other hand, as $a \neq c_{2}$, it follows by Lemma 3.1 that $a \not \leq h\left(c_{2}\right)$ which in turn implies $\left(a \circ c_{1}\right) \circ\left(a \circ c_{2}\right) \neq c_{2}$ because applying Lemma 3.3(ii) we have $a \leq h\left(\left(a \circ c_{1}\right) \circ\left(a \circ c_{2}\right)\right)$. Thus $\left(a \circ c_{1}\right) \circ\left(a \circ c_{2}\right) \unlhd a \circ c_{1}+c_{2}$ which means that $\left(a \circ c_{1}\right) \circ c_{2}$ is defined.

Now, assume that the lemma is true for each sequence of length $n$, and let $c_{1}, \ldots, c_{n+1}$ be a sequence in $A(L)$ with the properties (i) and (ii). By the induction hypothesis, $a \circ c_{1}\left(=b_{1}\right),\left(a \circ c_{1}\right) \circ c_{2}\left(=b_{2}\right), \ldots,\left(\ldots\left(a \circ c_{1}\right) \circ c_{2} \ldots\right) \circ$ $c_{n}\left(=b_{n}\right)$ and $a \circ c_{n+1}$ are all defined. Hence, by Lemma 4.1(i) and (iii), so are $b_{1} \circ\left(a \circ c_{n+1}\right), b_{2} \circ\left(b_{1} \circ\left(a \circ c_{n+1}\right)\right), \ldots, b_{n} \circ\left(b_{n-1} \circ \ldots\left(b_{2} \circ\left(b_{1} \circ\left(a \circ c_{n+1}\right)\right)\right)\right)$. Applying Lemma 3.3(ii) to the last expression we obtain

$$
h\left(b_{n} \circ\left(b_{n-1} \circ \ldots\left(b_{2} \circ\left(b_{1} \circ\left(a \circ c_{n+1}\right)\right)\right) \ldots\right)\right)=h\left(b_{n}+c_{n+1}\right) .
$$

As $c_{n+1} \not \leq h\left(a+c_{1}+\ldots+c_{n}\right)=h\left(b_{n}\right)$ and

$$
c_{n+1} \leq h\left(b_{n} \circ\left(b_{n-1} \circ \ldots\left(b_{2} \circ\left(b_{1} \circ\left(a \circ c_{n+1}\right)\right)\right) \ldots\right)\right),
$$

we have $b_{n} \neq b_{n} \circ\left(b_{n-1} \circ \ldots\left(b_{2} \circ\left(b_{1} \circ\left(a \circ c_{n+1}\right)\right)\right) \ldots\right)$. On the other hand, as $a \not \leq h\left(c_{n+1}\right)$ (use (i) and Lemma 3.1) and

$$
a \leq h\left(b_{n} \circ\left(b_{n-1} \circ \ldots\left(b_{2} \circ\left(b_{1} \circ\left(a \circ c_{n+1}\right)\right)\right) \ldots\right)\right),
$$

we obtain $c_{n+1} \neq b_{n} \circ\left(b_{n-1} \circ \ldots\left(b_{2} \circ\left(b_{1} \circ\left(a \circ c_{n+1}\right)\right)\right) \ldots\right)$. Thus

$$
b_{n} \circ\left(b_{n-1} \circ \ldots\left(b_{2} \circ\left(b_{1} \circ\left(a \circ c_{n+1}\right)\right)\right) \ldots\right) \unlhd b_{n}+c_{n+1}
$$

which yields that $b_{n} \circ c_{n+1}$ is defined and so is $\left(\ldots\left(\left(a \circ c_{1}\right) \circ c_{2}\right) \ldots\right) \circ c_{n+1}$. 
For a subset $A$ of $A(L)$, we denote by $[A]$ the least relative partial subsemilattice of $(A(L) ; \circ)$ containing $A$.

Lemma 4.3. Let $a \in A(L)$ and $A$ be a subset of $A(L)$ whose elements $b$ satisfy $a \not b$. Then the set $B$ of all $c \in[A \cup\{a\}]$ with $c \sqsubseteq$ a has a smallest element $d$ with respect to $\sqsubseteq$. Moreover, if $c \in B$ and $c \sqsubseteq b$ for all $b \in A$ then $c=d$.

Proof. If $A=\emptyset$ then $d=a$. So, assume that $A \neq \emptyset$. Pick $c_{1} \in A$. As $a \nsim c_{1}$, by Lemma 3.1 it follows that $a \circ c_{1}$ is defined and, by Lemma 3.3(ii), $a \circ c_{1} \in B$. Next, pick $c_{2} \in A$ such that $c_{2} \not \leq h\left(a+c_{1}\right)$. By Lemma 4.2, $\left(a \circ c_{1}\right) \circ c_{2}$ is defined and, by Lemma 3.3(ii), $\left(a \circ c_{1}\right) \circ c_{2} \in B$. Further, pick $c_{3} \in A$ such that $c_{3} \not \leq h\left(a+c_{1}+c_{2}\right)$. Again $\left(\left(a \circ c_{1}\right) \circ c_{2}\right) \circ c_{3}$ is defined and $\left(\left(a \circ c_{1}\right) \circ c_{2}\right) \circ c_{3} \in B$. Continuing this process we find, in a finite number of steps (recall that $L$ is finite), an element $d$ of $B$ such that $a \leq h(d)$ and $b \leq h(d)$ for all $b \in A$, that is, $d \sqsubseteq a$ and $d \sqsubseteq b$ for all $b \in A$.

Let $x \in B$. Then $x=p(X)$ for some semilattice term $p$ and $X \subseteq A \cup\{a\}$. Applying Lemma 3.3(ii) we have $h(x)=h\left(\sum X\right)$ where $\sum X$ is the join of $X$ in $L$. As $d \sqsubseteq a$ and $d \sqsubseteq b$ for all $b \in A$, we have $h\left(\sum X\right) \leq h(d)$ and hence $d \sqsubseteq x$, proving that $d$ is the smallest element of $(B, \sqsubseteq)$.

Now, assume that $c \in B$ and $c \sqsubseteq b$ for all $b \in A$. Notice that $c \sqsubseteq a$. So, applying Lemma 3.3(ii) and the definition of $d$, we obtain $c \sqsubseteq d$. Evidently, $d \sqsubseteq c$. Thus $c=d$.

Lemma 4.4. Let $\left(a_{0}, b\right){ }^{c_{0}} \nearrow\left(a_{1}, b\right){ }^{c_{1}} \nearrow \ldots{ }^{c_{n-1}} \nearrow\left(a_{n}, b\right)$ be a left descent in $L$ from $(a, b)$ with $n \geq 1$ and let $d$ be an atom of $L$ with $d \neq a$ and $d \sim b$. Then $a_{n} \sqsubseteq d$ and if $a_{1} \nsubseteq d$ then $n \geq 2$ and $a_{i} \circ d$ is defined for all $i<l$, where $l$ is the greatest number with $1 \leq l \leq n-1$ and $a_{l} \nsubseteq d$.

Proof. It is obvious that $a_{n} \sqsubseteq a\left(=a_{0}\right)$. Hence $d \sqsubseteq a_{n}$ implies $d \sqsubseteq a$ which contradicts $d \not a$ (see Lemma 3.1). So in order to prove that $a_{n} \sqsubseteq d$ we need only show that $a_{n}$ and $d$ are $\sqsubseteq$-comparable. Suppose they are not. Since $d \not a_{0}(=a)$, it follows that $d \circ a_{0}$ is defined and, by Lemma 4.1(i), $\left(d \circ a_{0}\right) \circ a_{0}=d \circ a_{0}$. Recalling the definition of a left descent we have $a_{1} \unlhd a_{0}+c_{0}$. So $a_{1} \circ a_{0}$ is defined and $a_{1} \circ a_{0}=a_{1}$. Hence, by Lemma 4.1(iii), $\left(d \circ a_{0}\right) \circ a_{1}$ is defined and, by Lemma 4.1 (i), $\left(\left(d \circ a_{0}\right) \circ a_{1}\right) \circ a_{1}=\left(d \circ a_{0}\right) \circ a_{1}$. But, as $a_{2} \unlhd a_{1}+c_{1}, a_{2} \circ a_{1}$ is defined and $a_{2} \circ a_{1}=a_{2}$. So, applying Lemma 4.1(iii) again we find that $\left(\left(d \circ a_{0}\right) \circ a_{1}\right) \circ a_{2}$ is defined and, by Lemma 4.1(i), $\left(\left(\left(d \circ a_{0}\right) \circ a_{1}\right) \circ a_{2}\right) \circ a_{2}=\left(\left(d \circ a_{0}\right) \circ a_{1}\right) \circ a_{2}$. Continuing this argument we conclude that $\left(\ldots\left(\left(d \circ a_{0}\right) \circ a_{1}\right) \circ a_{2} \ldots\right) \circ a_{n}(=f)$ is defined. Now notice that, by Lemma 3.3(ii) and the property that $a_{n} \sqsubseteq a_{i}$ for all $i<n$, we get $h(f)=h\left(d+a_{n}\right)$. Thus, by Lemma 3.3(i), $f \leq d+a_{n}$. Hence, by Lemma 3.1, either $f=d$, or $f=a_{n}$, or $f \unlhd d+a_{n}$. If $f=d$ then, as $a_{n} \leq h(f)$ (use Lemma 3.3(ii)), we obtain $a_{n} \leq h(d)$, that is, $d \sqsubseteq a_{n}$ which 
contradicts our assumption. If $f=a_{n}$ then, as $d \leq h(f)$ (use Lemma 3.3(ii)), we have $d \leq h\left(a_{n}\right)$, that is, $a_{n} \sqsubseteq d$ which again contradicts our assumption. If $f \unlhd d+a_{n}$ then $d \nsim a_{n}$ which together with the assumption that $d \sim b$ contradicts the assumption that $\left(a_{0}, b\right), \ldots,\left(a_{n}, b\right)$ is a left descent from $(a, b)$. Thus $d$ and $a_{n}$ are $\sqsubseteq$-comparable. Consequently, $a_{n} \sqsubseteq d$.

We now show that $a_{i} \circ d$ is defined for all $i \leq l$ provided that $a_{1} \nsubseteq d$. That $a_{0} \circ d$ is defined is obvious since $a_{0}=a$ and $d \nsim a$. Fix $i=1, \ldots, l$. Arguing as above one shows that $\left(\ldots\left(d \circ a_{0}\right) \circ a_{1} \ldots\right) \circ a_{i}$ is defined. Since $a_{i} \sqsubseteq a_{k}$ for all $k=0, \ldots, i$, by Lemma 3.3(ii) we have $h\left(d+a_{i}\right)=h((\ldots)(d \circ$ $\left.\left.\left.a_{0}\right) \circ a_{1} \ldots\right) \circ a_{i}\right)$. Hence, by Lemma 3.3(i), $\left(\ldots\left(d \circ a_{0}\right) \circ a_{1} \ldots\right) \circ a_{i} \leq a_{i}+d$. If $\left(\ldots\left(d \circ a_{0}\right) \circ a_{1} \ldots\right) \circ a_{i}=a_{i}$ then $a_{i} \sqsubseteq d$, a contradiction since $i \leq l$ and, therefore, $a_{i} \nsubseteq d$. If $\left(\left(d \circ a_{0}\right) \circ a_{1} \ldots\right) \circ a_{i}=d$ then $d \sqsubseteq a_{0}$ which in view of Lemma 3.1 contradicts $d \not a_{0}(=a)$. Thus $\left(\left(d \circ a_{0}\right) \circ a_{1} \ldots\right) \circ a_{i} \unlhd a_{i}+d$ and, therefore, $a_{i} \circ d$ is defined which completes the proof.

Lemma 4.5. Let $\left(a_{0}, b\right){ }^{c_{0}} \nearrow\left(a_{1}, b\right){ }^{c_{1}} \nearrow \ldots{ }^{c_{n-1}} \nearrow\left(a_{n}, b\right)$ be a left descent in $L$ from $(a, b)$ with $n \geq 1$ and let $d$ be an atom of $L$ with $d \ngtr a, d \sim b$ and $a_{1} \nsubseteq d$. Then there exists a left descent in $L$ from $(a, b)$ which ends in $\left(a_{n}, b\right)$ and starts with $\left(a_{0}, b\right){ }^{c_{0}} \nearrow\left(a_{1}, b\right) d \nearrow\left(a_{1} \circ d, b\right)$.

Proof. By Lemma 4.4, $a_{i} \circ d$ is defined for each $i=0,1, \ldots, l$, where $l$ is the greatest number such that $1 \leq l \leq n-1$ and $a_{l} \nsubseteq d$. In particular, $a_{1} \circ d$ is defined which, by Lemma 3.3, implies $a_{1} \circ d \leq a_{1}+d$ and so, by Lemma 3.1, we have either $a_{1} \circ d \in\left\{a_{1}, d\right\}$ or $a_{1} \circ d \unlhd a_{1}+d$. If $a_{1} \circ d=a_{1}$ then $a_{1} \sqsubseteq d$, a contradiction. If $a_{1} \circ d=d$ then $d \sqsubseteq a_{1}$ and hence $d \sqsubseteq a_{0}$, since $a_{1} \sqsubseteq a_{0}$, but this in view of Lemma 3.1 contradicts $d \neq a_{0}$. Thus $a_{1} \circ d \unlhd a_{1}+d$. So we have a sequence

$$
\left(a_{0}, b\right)^{c_{0}} \nearrow\left(a_{1}, b\right)^{d} \nearrow\left(a_{1} \circ d, b\right) .
$$

Cas e 1: $l=1$. If $a_{2}=a_{1} \circ d$ then the proof is complete. So, consider the case when $a_{2} \neq a_{1} \circ d$. As $a_{2} \sqsubseteq d$ because $l=1$ and, as $a_{2} \sqsubseteq a_{1}$ and $a_{1} \circ d$ is defined, by Lemma 3.3(ii), we obtain $a_{2} \sqsubseteq a_{1} \circ d \sqsubseteq a_{1}$. This, by $a_{2}=a_{1} \circ c_{1}$ and Lemma 4.1(ii), implies that $\left(a_{1} \circ d\right) \circ c_{1}$ is defined and $\left(a_{1} \circ d\right) \circ c_{1}=a_{2}$. So, by Lemma 3.3(i), we have $a_{2} \leq a_{1} \circ d+c_{1}$. Hence $a_{2} \unlhd a_{1} \circ d+c_{1}$ because $a_{2} \neq a_{1} \circ d$ and $a_{2} \neq c_{1}$. So, the desired descent is

$$
\left(a_{0}, b\right)^{c_{0}} \nearrow\left(a_{1}, b\right)^{d} \nearrow\left(a_{1} \circ d, b\right)^{c_{1}} \nearrow\left(a_{2}, b\right)^{c_{2}} \nearrow \ldots{ }^{c_{n-1}} \nearrow\left(a_{n}, b\right) \text {. }
$$

Case 2: $l \geq 2$. For a fixed $1 \leq i \leq l-1$ we have

Claim 1. If $c_{i} \nsim a_{i} \circ d$ then $\left(a_{i} \circ d, b\right){ }^{c_{i}} \nearrow\left(a_{i+1} \circ d, b\right)$.

Proof. As $a_{i} \circ d$ is defined and, since $c_{i} \not a_{i} \circ d$, so is $c_{i} \circ\left(a_{i} \circ d\right)$, applying Proposition 3.4 we have $a_{i+1} \circ d=\left(c_{i} \circ a_{i}\right) \circ d=c_{i} \circ\left(a_{i} \circ d\right)$. So, as $c_{i} \nsim a_{i} \circ d$, it follows that $a_{i+1} \circ d \unlhd a_{i} \circ d+c_{i}$ which means that $\left(a_{i} \circ d, b\right){ }^{c_{i}} \nearrow\left(a_{i+1} \circ d, b\right)$. 
Claim 2. If $c_{i} \sim a_{i} \circ d$ then $a_{i} \circ d=a_{i+1} \circ d$.

Proof. As $a_{i} \circ d$ is defined and $a_{i} \circ a_{i+1}=a_{i+1}$ (recall that $a_{i+1} \unlhd a_{i}+c_{i}$ ), it follows by Lemma 4.1(i) and (iii) that $\left(a_{i} \circ d\right) \circ a_{i+1}$ is defined. Applying Lemma 3.3(ii) we have $h\left(\left(a_{i} \circ d\right) \circ a_{i+1}\right)=h\left(a_{i} \circ d+a_{i+1}\right)$. We now show that $a_{i} \circ d \sqsubseteq c_{i}$. Suppose otherwise, that is, $c_{i} \not \leq h\left(a_{i} \circ d\right)$. As $a_{i} \circ d \sim c_{i}, c_{i} \leq$ $h\left(\left(a_{i} \circ d\right) \circ a_{i+1}\right)$, since $c_{i} \leq h\left(a_{i+1}\right)$, and $h\left(\left(a_{i} \circ d\right) \circ a_{i+1}\right)=h\left(a_{i} \circ d+a_{i+1}\right)$, by (h8) it follows that $h\left(\left(a_{i} \circ d\right) \circ a_{i+1}\right)=h\left(c_{i}+a_{i+1}\right)=h\left(a_{i+1}\right)$. So, by Lemma 3.3(ii), we obtain $d \leq h\left(a_{i+1}\right)$, a contradiction since $i+1 \leq l$ and, therefore, $a_{i+1} \nsubseteq d$. Thus $a_{i} \circ d \sqsubseteq c_{i}$. So, applying Lemma 3.3(ii) again, we obtain

$$
\begin{aligned}
h\left(a_{i} \circ d\right) & =h\left(a_{i} \circ d+c_{i}\right)=h\left(a_{i}+d+c_{i}\right)=h\left(a_{i}+c_{i}+d\right) \\
& =h\left(a_{i+1}+d\right)=h\left(a_{i+1} \circ d\right) .
\end{aligned}
$$

Hence, by Lemma 3.3(i), $a_{i} \circ d=a_{i+1} \circ d$ which shows the claim.

Applying Claims 1 and 2 we get a sequence

$$
\left(a_{0}, b\right)^{c_{0}} \nearrow\left(a_{1}, b\right)^{d} \nearrow\left(a_{1} \circ d, b\right) \nearrow \ldots \nearrow\left(a_{l} \circ d, b\right) .
$$

Now, we split Case 2 into the following two subcases.

$\mathrm{Subcase} \mathrm{A}: a_{l} \circ d=a_{l+1}$. The desired left descent is

$$
\left(a_{0}, b\right)^{c_{0}} \nearrow\left(a_{1}, b\right)^{d} \nearrow\left(a_{1} \circ d, b\right) \nearrow \ldots \nearrow\left(a_{l} \circ d, b\right)^{c_{l+1}} \nearrow \ldots{ }^{c_{n-1}} \nearrow\left(a_{n}, b\right) .
$$

Subcase B: $a_{l} \circ d \neq a_{l+1}$. As $a_{l+1} \sqsubseteq a_{l}$ and $a_{l+1} \sqsubseteq d$, we have $a_{l+1} \sqsubseteq a_{l} \circ d \sqsubseteq a_{l}$. So, as $a_{l+1}=a_{l} \circ c_{l}$, by Lemma 4.1(ii) we see that $\left(a_{l} \circ d\right) \circ c_{l}$ is defined and $\left(a_{l} \circ d\right) \circ c_{l}=a_{l+1}$. As $a_{l+1} \notin\left\{a_{l} \circ d, c_{l}\right\}$, applying Lemmas 3.3(i) and 3.1 we obtain $a_{l+1} \unlhd\left(a_{l} \circ d\right)+c_{l}$. So the desired left descent from $(a, b)$ is

$$
\begin{aligned}
&\left(a_{0}, b\right)^{c_{0}} \nearrow\left(a_{1}, b\right)^{d} \nearrow\left(a_{1} \circ d, b\right) \nearrow \ldots \\
& \ldots \nearrow\left(a_{l} \circ d, b\right)^{c_{l}} \nearrow\left(a_{i+1}, b\right)^{c^{c_{i+1}} \nearrow \ldots{ }^{c_{n-1}} \nearrow\left(a_{n}, b\right) .}
\end{aligned}
$$

This completes the proof of the lemma.

We are now ready to prove the main lemma of this section.

Lemma 4.6. Suppose $L$ is a finite atomistic lattice that admits an equaclosure operator. Then $L$ has univocally terminating left descents.

Proof. Let $(a, b)$ be a pair of atoms of $L$ with $a \sim b$. Let

$$
\left(a_{0}, b\right)^{c_{0}} \nearrow \ldots{ }^{c_{n-1}} \nearrow\left(a_{n}, b\right)
$$

be a left descent in $L$ from $(a, b)$. Of course, we may assume that $n \geq 1$ since otherwise the pair $(a, b)$ would be free and this would mean that any left descent from $(a, b)$ consists only of $(a, b)$. The assumption gives that $x \nsim a$ and $x \sim b$ for some $x$ of $A(L)$. 
We define inductively two sequences $A_{0}, A_{1}, \ldots, B_{0}, B_{1}, \ldots$ of non-empty subsets of $A(L)$ and a sequence $d_{0}, d_{1}, \ldots$ in $A(L)$. Recall that, for $C \subseteq$ $A(L),[C]$ denotes the least relative partial subsemilattice of $(A(L) ; \circ)$ containing $C$. We set

$$
\begin{aligned}
& A_{0}:=\{x \in A(L): x \nsim a \text { and } x \sim b\}, \\
& B_{0}:=\left\{x \in\left[A_{0} \cup\{a\}\right]: x \sqsubseteq a\right\}, \\
& d_{0}:=\text { the smallest element of }\left(B_{0}, \sqsubseteq\right)
\end{aligned}
$$

(which exists in view of Lemma 4.3). Assume now that $A_{i}, B_{i}$ and $d_{i}$ are defined. If there is no $x$ in $A(L)$ such that $x \nsim d_{i}$ and $x \sim b$, then we stop the construction. Otherwise we set

$$
\begin{aligned}
A_{i+1} & :=\left\{x \in A(L): x \not d_{i} \text { and } x \sim b\right\}, \\
B_{i+1} & :=\left\{x \in\left[A_{i+1} \cup\left\{d_{i}\right\}\right]: x \sqsubseteq d_{i}\right\}, \\
d_{i+1} & :=\text { the smallest element of }\left(B_{i+1}, \sqsubseteq\right)
\end{aligned}
$$

(which exists in view of Lemma 4.3). As $L$ is finite, the above procedure terminates after a finite number of steps, say $k$. Thus we have sequences $A_{0}, A_{1}, \ldots, A_{k}, B_{0}, B_{1}, \ldots, B_{k}$ and $d_{0}, d_{1}, \ldots, d_{k}$.

The idea of the proof is to construct a left descent in $L$ from $(a, b)$ that ends in $\left(a_{n}, b\right)$ and contains each of the pairs $\left(d_{0}, b\right), \ldots,\left(d_{k}, b\right)$. This will give that $\left(d_{k}, b\right)=\left(a_{n}, b\right)$, that is, $d_{k}=a_{n}$ since, for each $x$ in $A(L), x \sim b$ implies $x \sim d_{k}$. This in turn will show that $L$ has univocally terminating left descents because the sequence $d_{0}, d_{1}, \ldots, d_{k}$ is uniquely determined by the pair $(a, b)$.

We first construct a left descent in $L$ from $(a, b)$ that ends in $\left(a_{n}, b\right)$ and contains $\left(d_{0}, b\right)$.

Case 1: For each $x$ in $A_{0}, a_{1} \sqsubseteq x$. Notice that $a_{1} \in B_{0}$ since $c_{0} \in A_{0}$ and $a_{1}=a \circ c_{0}$. So $a_{1}=d_{0}$ by Lemma 4.3. Thus $\left(d_{0}, b\right)$ already occurs in $(D 1)$.

Case 2: $a_{1} \nsubseteq x$ for some $x$ of $A_{0}$. Pick $e_{1}$ in $A_{0}$ such that $a_{1} \nsubseteq e_{1}$. Then Lemma 4.5 yields a left descent in $L$ from $(a, b)$

$$
\left(a_{0}, b\right){ }^{c_{0}} \nearrow\left(a_{1}, b\right){ }^{e_{1}} \nearrow\left(a_{1} \circ e_{1}, b\right) \nearrow \ldots \nearrow\left(a_{n}, b\right) .
$$

If there is no $x$ in $A_{0}$ with $a_{1} \circ e_{1} \nsubseteq x$ then, by Lemma 4.3, $a_{1} \circ e_{1}=d_{0}$ and hence $(D 2)$ contains the pair $\left(d_{0}, b\right)$. Otherwise, pick $e_{2}$ in $A_{0}$ with $a_{1} \circ e_{1} \nsubseteq e_{2}$. Obviously, $e_{2} \sim b$ since $e_{2} \in A_{0}$. We show $e_{2} \nsim a_{1}$. This, by $a_{1} \circ e_{1} \nsubseteq e_{2}$, will allow us to apply Lemma 4.5 to the left descent

$$
\left(a_{1}, b\right){ }^{e_{1}} \nearrow\left(a_{1} \circ e_{1}, b\right) \nearrow \ldots \nearrow\left(a_{n}, b\right)
$$

obtained from $(D 2)$ by cancelling $\left(a_{0}, b\right)$. We will then have a left descent from $\left(a_{1}, b\right)$ that ends in $\left(a_{n}, b\right)$ and whose first three elements are $\left(a_{1}, b\right){ }^{e_{1}} \nearrow$ 
$\left(a_{1} \circ e_{1}, b\right){ }^{e_{2}} \nearrow\left(\left(a_{1} \circ e_{1}\right) \circ e_{2}, b\right)$. This in turn will give us a left descent from $(a, b)$ that ends in $\left(a_{n}, b\right)$ and whose first four elements are

$$
\left(a_{0}, b\right)^{c_{0}} \nearrow\left(a_{1}, b\right){ }^{e_{1}} \nearrow\left(a_{1} \circ e_{1}, b\right){ }^{e_{2}} \nearrow\left(\left(a_{1} \circ e_{1}\right) \circ e_{2}, b\right) .
$$

So we need to show that $e_{2} \nsim a_{1}$. Since $a_{1} \circ e_{1} \sqsubseteq a_{1}$ and $a_{1} \circ e_{1} \nsubseteq e_{2}$, we have $a_{1} \nsubseteq e_{2}$. By Lemma 4.4, $a_{1} \circ e_{2}$ is defined. Hence $a_{1} \circ e_{2} \leq a_{1}+e_{2}$. If $a_{1} \circ e_{2}=a_{1}$ then $a_{1} \sqsubseteq e_{2}$ which is impossible since $a_{1} \circ e_{1} \sqsubseteq a_{1}$ and $a_{1} \circ e_{1} \nsubseteq e_{2}$. If $a_{1} \circ e_{2}=e_{2}$ then $e_{2} \sqsubseteq a_{1} \sqsubseteq a_{0}(=a)$ which is impossible since $e_{2} \in A_{0}$ and so $e_{2} \not \alpha a$ (use Lemma 3.1). Thus $a_{1} \circ e_{2} \unlhd a_{1}+e_{2}$ which implies that $e_{2} \nsim a_{1}$.

Now, if $\left(a_{1} \circ e_{1}\right) \circ e_{2} \sqsubseteq x$ for all $x \in A_{0}$ then, by Lemma $4.3,\left(a_{1} \circ e_{1}\right) \circ$ $e_{2}=d_{0}$ and the construction of a left descent in $L$ from $(a, b)$ that ends in $\left(a_{n}, b\right)$ and contains $\left(d_{0}, b\right)$ is complete. Otherwise we pick $e_{3}$ in $A_{0}$ with $\left(a_{1} \circ e_{1}\right) \circ e_{2} \nsubseteq e_{3}$. Recall that we have already found a left descent in $L$ from $\left(a_{0}, b\right)$ that ends in $\left(a_{n}, b\right)$ and starts with $(D 4)$.

We show $a_{1} \circ e_{1} \nsim e_{3}$. As $\left(a_{1} \circ e_{1}\right) \circ e_{2} \sqsubseteq a_{1}$ and $\left(a_{1} \circ e_{1}\right) \circ e_{2} \nsubseteq e_{3}$, we have $a_{1} \nsubseteq e_{3}$. So, by Lemma 4.4, $\left(a_{1} \circ e_{1}\right) \circ e_{3}$ is defined. Hence $\left(a_{1} \circ e_{1}\right) \circ e_{3} \leq a_{1} \circ e_{1}+e_{3}$. If $\left(a_{1} \circ e_{1}\right) \circ e_{3}=a_{1} \circ e_{1}$ then $a_{1} \circ e_{1} \sqsubseteq e_{3}$ which, by $\left(a_{1} \circ e_{1}\right) \circ e_{2} \sqsubseteq a_{1} \circ e_{1}$, implies $\left(a_{1} \circ e_{1}\right) \circ e_{2} \sqsubseteq e_{3}$, a contradiction. If $\left(a_{1} \circ e_{1}\right) \circ e_{3}=e_{3}$ then $e_{3} \sqsubseteq a_{1} \circ e_{1} \sqsubseteq a_{1} \sqsubseteq a$, a contradiction since $e_{3} \in A_{0}$ and, by Lemma 3.1, $e_{3} \nsubseteq a$. Thus $\left(a_{1} \circ e_{1}\right) \circ e_{3} \unlhd a_{1} \circ e_{1}+e_{3}$ which implies that $a_{1} \circ e_{1} \not e_{3}$.

Notice that $e_{3} \sim b$ since $e_{3} \in A_{0}$. Hence applying Lemma 4.5 we have a left descent in $L$ from $\left(a_{1} \circ e_{1}, b\right)$ that ends in $\left(a_{n}, b\right)$ and starts with

$$
\left(a_{1} \circ e_{1}, b\right) e^{e_{2}} \nearrow\left(\left(a_{1} \circ e_{1}\right) \circ e_{2}, b\right) e^{e_{3}} \nearrow\left(\left(\left(a_{1} \circ e_{1}\right) \circ e_{2}\right) \circ e_{3}, b\right) .
$$

So we have a left descent in $L$ from $(a, b)$ that ends in $\left(a_{n}, b\right)$ and starts with $\left(a_{0}, b\right){ }^{c_{0}} \nearrow\left(a_{1}, b\right){ }^{e_{1}} \nearrow\left(a_{1} \circ e_{1}, b\right){ }^{e_{2}} \nearrow\left(\left(a_{1} \circ e_{1}\right) \circ e_{2}, b\right){ }^{e_{3}} \nearrow\left(\left(\left(a_{1} \circ e_{1}\right) \circ e_{2}\right) \circ e_{3}, b\right)$.

If, for each $x$ of $A_{0},\left(\left(a_{1} \circ e_{1}\right) \circ e_{2}\right) \circ e_{3} \sqsubseteq x$ then, by Lemma $4.3,\left(\left(a_{1} \circ e_{1}\right) \circ\right.$ $\left.e_{2}\right) \circ e_{3}=d_{0}$ and the construction of a left descent in $L$ from $(a, b)$ that ends in $\left(a_{n}, b\right)$ and contains $\left(d_{0}, b\right)$ is complete. Otherwise we pick $e_{4}$ in $A_{0}$ with $\left(\left(a_{1} \circ e_{1}\right) \circ e_{2}\right) \circ e_{3} \nsubseteq e_{4}$ and continue the above argument. As $A_{0}$ is finite, after a finite number of steps we find a left descent

$$
\left(a_{0}, b\right) \nearrow \ldots \nearrow\left(d_{0}, b\right) \nearrow \ldots \nearrow\left(a_{n}, b\right)
$$

that ends in $\left(a_{n}, b\right)$ and contains the pair $\left(d_{0}, b\right)$. Now applying the above arguments to the part of the resulting left descent that begins with $\left(d_{0}, b\right)$ we find a left descent that ends in $\left(a_{n}, b\right)$ and contains $\left(d_{1}, b\right)$. Next gluing the left descent from $\left(d_{0}, b\right)$ obtained in this way with the beginning part of the previously constructed left descent we obtain a left descent from $(a, b)$ that ends in $\left(a_{n}, b\right)$ and contains both $\left(d_{0}, b\right)$ and $\left(d_{1}, b\right)$. Continuing in this 
way we find a left descent in $L$ from $(a, b)$ that ends in $\left(a_{n}, b\right)$ and contains all the pairs $\left(d_{m}, b\right)$, where $m=0, \ldots, k$. This completes the proof.

5. Parities of right and left slaloms. In this section we show that in every finite atomistic lattice that admits an equa-closure operator right and left slaloms have different parities.

As in the previous section $L$ is a finite atomistic lattice with an equaclosure operator $h$.

Lemma 5.1. If $a \sim b$ and $\left(a_{0}, b\right){ }^{c_{0}} \nearrow \ldots{ }^{c_{n-1}} \nearrow\left(a_{n}, b\right)$ is a left descent in $L$ from $(a, b)$ with $a_{n}$ and $b \sqsubseteq$-comparable then $a_{0} \sqsubseteq b$ or $b \sqsubseteq a_{n}$.

Proof. Assume $b \nsubseteq a_{n}$. Then $a_{n} \sqsubseteq b$ since $a_{n}$ and $b$ are $\sqsubseteq$-comparable. Denote by $k$ the smallest number $0 \leq k \leq n$ such that $a_{k} \sqsubseteq b$. Since $a_{n} \sqsubseteq b$, such a number exists. We show $k=0$ which will complete the proof. Suppose $k \geq 1$. We have

Claim. For each $i=0, \ldots, n, a_{i} \sqsubseteq b$ or $b \sqsubseteq a_{i}$.

Proof. We split the proof into two cases.

Case 1: $a_{k-1} \circ b$ is not defined. Then $a_{k-1} \sim b$. So, as $a_{k-1} \nsubseteq b$, $a_{k} \sqsubseteq b$ and $h\left(a_{k}\right)=h\left(a_{k-1}+c_{k-1}\right)$, by (h8) we have $h\left(a_{k}\right)=h\left(b+c_{k-1}\right)$. This implies $a_{k} \leq b+c_{k-1}$. But $b \sim c_{k-1}$. So $a_{k}=b$; the case $a_{k}=c_{k-1}$ is impossible since $a_{k} \unlhd a_{k-1}+c_{k-1}$. Hence, for each $0 \leq i \leq n, a_{i} \sqsubseteq b$ or $b \sqsubseteq a_{i}$ since $a_{n} \sqsubseteq \ldots \sqsubseteq a_{0}$.

Case 2: $a_{k-1} \circ b$ is defined. We first show $b \sqsubseteq a_{0}$. Suppose $b \nsubseteq a_{0}$. Then, as $b \sim a_{0}$ because $b \sim a$ and $a_{0}=a, a_{0} \leq h\left(a_{k-1} \circ b\right)$ because $a_{0} \leq h\left(a_{k-1}\right)$, and, by Lemma 3.3(ii), $h\left(a_{k-1} \circ b\right)=h\left(a_{k-1}+b\right)$, it follows by (h8) that $h\left(a_{k-1} \circ b\right)=h\left(a_{k-1}+a_{0}\right)$ which, by $a_{0} \leq h\left(a_{k-1}\right)$, implies $h\left(a_{k-1} \circ b\right)=h\left(a_{k-1}\right)$. So, applying (h5) we obtain $a_{k-1} \circ b=a_{k-1}$. This, however, by Lemma 3.3(ii), yields $a_{k-1} \sqsubseteq b$ which contradicts the minimality of $k$. Thus $b \sqsubseteq a_{0}$.

As $a_{k-1} \circ b$ is defined, we have either $a_{k-1} \circ b \unlhd a_{k-1}+b$ or $a_{k-1} \circ b \in$ $\left\{a_{k-1}, b\right\}$. Accordingly we consider two subcases.

Subcase A: $a_{k-1} \circ b \unlhd a_{k-1}+b$. As $a_{0} \sim b$, it follows that $k-1 \neq 0$, that is, $k \geq 2$. We show that $b \sqsubseteq c_{i}$ for all $i<k-1$. Suppose $b \nsubseteq c_{i}$ for some $i<k-1$. Then $c_{i} \not \leq h(b)$. As $b \sim c_{i}$ and $c_{i} \leq h\left(a_{k-1} \circ b\right)$ because $c_{i} \leq h\left(a_{k-1}\right)$ and, by Lemma 3.3(ii), $h\left(a_{k-1} \circ b\right)=h\left(a_{k-1}+b\right)$, it follows by (h8) that $h\left(a_{k-1} \circ b\right)=h\left(a_{k-1}+c_{i}\right)$. But $c_{i} \leq h\left(a_{k-1}\right)$. So $h\left(a_{k-1} \circ b\right)=h\left(a_{k-1}\right)$. Therefore, by (h5), $a_{k-1} \circ b=a_{k-1}$ which yields $a_{k-1} \sqsubseteq b$ and contradicts the minimality of $k$. Thus $b \sqsubseteq c_{i}$ for all $i<k-1$. In particular, $b \sqsubseteq c_{0}$. So, as $b \sqsubseteq a_{0}$ (see above), we obtain $a_{0} \circ c_{0} \leq a_{0}+c_{0} \leq h(b)$ which, by $a_{1}=a_{0} \circ c_{0}$, implies $b \sqsubseteq a_{1}$. But $b \sqsubseteq c_{1}$ (if 
$k-1 \geq 2$ ). So, as $b \sqsubseteq a_{1}$, we obtain as before $b \sqsubseteq a_{2}$; recall that $a_{2}=a_{1} \circ c_{1}$. Eventually, we get $b \sqsubseteq a_{k-1}$. Thus $a_{k} \sqsubseteq b \sqsubseteq a_{k-1}$, and, therefore, $a_{i} \sqsubseteq b$ or $b \sqsubseteq a_{i}$ for all $i=0, \ldots, n$.

Subcase B: $a_{k-1} \circ b \in\left\{a_{k-1}, b\right\}$. Then $a_{k-1} \circ b=b$ because the case $a_{k-1} \circ b=a_{k-1}$ contradicts the minimality of $k$. Hence $b \sqsubseteq a_{k-1}$ and so $a_{k} \sqsubseteq b \sqsubseteq a_{k-1}$. Thus either $a_{i} \sqsubseteq b$ or $b \sqsubseteq a_{i}$ for all $i=0, \ldots, n$.

Thus the proof of the claim is complete.

The claim, definition of $k$ and $k \geq 1$ show that $a_{k} \sqsubseteq b \sqsubseteq a_{k-1}$. But $a_{k}=a_{k-1} \circ c_{k-1}$. So, by Lemma 4.1(ii), $b \circ c_{k-1}$ is defined and $b \circ c_{k-1}=a_{k}$. From the definition of a left descent it follows that $b \sim c_{k-1}$, and, therefore, we have $a_{k} \in\left\{b, c_{k-1}\right\}$. But $a_{k} \neq c_{k-1}$ because $a_{k} \unlhd a_{k-1}+c_{k-1}$. So $a_{k}=b$. Since $b \nsubseteq a_{n}$, this yields $k<n$. Hence $k+1 \leq n$ and, therefore, $a_{k+1} \unlhd a_{k}+c_{k}$. So, as $a_{k}=b$, we obtain $a_{k+1} \unlhd b+c_{k}$. But this is impossible because $b \sim c_{k}$. Thus $k=0$ which means $a_{0} \sqsubseteq b$ and completes the proof of the lemma.

Lemma 5.2. If $a \sim b$ and $\left(a, b_{0}\right) \searrow^{c_{0}} \ldots \searrow^{c_{n-1}}\left(a, b_{n}\right)$ is a right descent in $L$ from $(a, b)$ with $a$ and $b_{n} \sqsubseteq$-comparable then $b_{0} \sqsubseteq a$ or $a \sqsubseteq b_{n}$.

Proof. Notice that $\left(b_{0}, a\right){ }^{c_{0}} \nearrow \ldots{ }^{c_{n-1}} \nearrow\left(b_{n}, a\right)$ is a left descent in $L$ from $(b, a)$. Next apply Lemma 5.1.

Lemma 5.3. Suppose $a, b \in A(L), a \sim b$ and $\left(a_{0}, b\right){ }^{c_{0}} \nearrow \ldots{ }^{c_{n-1}} \nearrow$ $\left(a_{n}, b\right) \searrow^{d_{0}} \ldots \searrow^{d_{m-1}}\left(a_{n}, b_{m}\right)$ is a left descent followed by a right descent, both non-trivial, with $a_{0}=a$. Then the following conditions hold:

(i) $a_{n} \sqsubseteq b_{m}$ implies $a \sqsubseteq b$;

(ii) $a_{n} \neq b_{m}$ and $b_{m} \sqsubseteq a_{n}$ imply $b \sqsubseteq a$.

Proof. (i) Assume $a_{n} \sqsubseteq b_{m}$. As $\left(a_{n}, b\right) \searrow_{0}^{d_{0}} \ldots \searrow^{d_{m-1}}\left(a_{n}, b_{m}\right)$ is a right descent from $\left(a_{n}, b\right)$, we have $b_{m} \sqsubseteq b$ and hence $a_{n} \sqsubseteq b$. So, by Lemma 5.1, either $a \sqsubseteq b$ or $a_{n}=b$. On the other hand, as both descents are non-trivial, we have $a_{n} \neq b$. Thus $a \sqsubseteq b$.

(ii) Assume $a_{n} \neq b_{m}$ and $b_{m} \sqsubseteq a_{n}$. Applying Lemma 5.2 to the right descent $\left(a_{n}, b\right) \searrow_{0}^{d_{0}} \ldots \searrow^{d m-1}\left(a_{n}, b_{m}\right)$ we obtain $b \sqsubseteq a_{n}$. But $\left(a_{0}, b\right){ }^{c_{0}} \nearrow$ $\ldots{ }^{c_{n-1}} \nearrow\left(a_{n}, b\right)$ is a left descent from $(a, b)$. So $a_{n} \sqsubseteq a$. Hence $b \sqsubseteq a$.

Lemma 5.4. Suppose $a, b \in A(L), a \sim b$ and $\left(a_{0}, b\right) \searrow^{c_{0}} \ldots \searrow_{n-1}$ $\left(a_{0}, b_{n}\right){ }^{d_{0}} \nearrow \ldots{ }^{d_{m-1}} \nearrow\left(a_{m}, b_{n}\right)$ is a right descent followed by a left one, both non-trivial, with $a_{0}=a$. Then the following conditions hold:

(i) $b_{n} \sqsubseteq a_{m}$ implies $b \sqsubseteq a$;

(ii) $a_{m} \neq b_{n}$ and $a_{m} \sqsubseteq b_{n}$ implies $a \sqsubseteq b$.

Proof. Notice that $\left(b, a_{0}\right)^{c_{0}} \nearrow \ldots{ }^{c_{n-1}} \nearrow\left(b_{n}, a_{0}\right) \searrow^{d_{0}} \ldots \searrow^{d_{m-1}}$ $\left(b_{n}, a_{m}\right)$ satisfies the assumptions of Lemma 5.3. 
Lemma 5.5. If $a, b \in A(L), a \sim b$ and there exists a slalom in $L$ with the origin at $(a, b)$ that is left and exact then $a \sqsubseteq b$ whenever the slalom is even, and $b \sqsubseteq a$ whenever it is odd.

Pro of. Let $(a, b), \ldots,(c, c)$ be a relevant slalom and $k$ be the number of its descents.

We consider first the case $k=2$. Then the slalom is of the form $\left(a_{0}, b\right){ }^{c_{0}} \nearrow \ldots{ }^{c_{n-1}} \nearrow\left(a_{n}, b\right) \searrow_{0}^{d_{0}} \ldots \searrow^{d_{m-1}}\left(a_{n}, b_{m}\right)$ where $a_{0}=a$ and $\left(a_{n}, b_{m}\right)=(c, c)$. In particular, $a_{n}=b_{m}$. So applying Lemma 5.3 we obtain $a \sqsubseteq b$.

Assume that $k$ is even and $k \geq 4$. Consider the last two descents in our slalom. They form a left and exact slalom of the form

$$
\left(a_{0}^{\prime}, b^{\prime}\right) c_{0}^{\prime} \nearrow \ldots c_{n-1}^{\prime} \nearrow\left(a_{n}^{\prime}, b^{\prime}\right) \searrow_{0}^{\prime} \ldots \searrow_{m-1}^{\prime}\left(a_{n}^{\prime}, b_{m}^{\prime}\right)(=(c, c)) .
$$

Applying Lemma 5.3 to $(S)$, we obtain $a_{0}^{\prime} \sqsubseteq b^{\prime}$. Now notice that the part of our slalom without $(S)$ but with $\left(a_{0}^{\prime}, b^{\prime}\right)$ forms a left and even slalom that ends in $\left(a_{0}^{\prime}, b^{\prime}\right)$ and starts with $(a, b)$. So, as $a_{0}^{\prime} \sqsubseteq b^{\prime}$, applying Lemma 5.3 a suitable number of times we eventually obtain $a \sqsubseteq b$.

Now, let $k$ be odd. If $k=1$ then evidently $b \sqsubseteq a$. So, let $k \geq 3$. Consider the last two descents in $(a, b), \ldots,(c, c)$. They form a right and exact slalom of the form

$$
\left(a_{0}^{\prime}, b^{\prime}\right) \searrow_{0}^{c_{0}^{\prime}} \ldots \searrow_{n-1}^{\prime}\left(a_{n}^{\prime}, b\right) d_{0}^{\prime} \nearrow \ldots{ }^{d_{m-1}^{\prime}} \nearrow\left(a_{n}^{\prime}, b_{m}^{\prime}\right)(=(c, c)) .
$$

By Lemma 5.4, $b^{\prime} \sqsubseteq a_{0}^{\prime}$, so that repeated application of that lemma yields $b \sqsubseteq a$.

Again, we have a "dual" version:

Lemma 5.6. If $a, b \in A(L), a \sim b$ and there exists a slalom in $L$ with the origin at $(a, b)$ that is right and exact then $b \sqsubseteq a$ whenever the slalom is even, and $a \sqsubseteq b$ whenever it is odd.

P r o o f. Let $(a, b), \ldots,(c, d)$ be a relevant slalom. Then $(b, a), \ldots,(d, c)$ forms a slalom with the origin at $(b, a)$ that is left and exact. The lemma now follows from Lemma 5.5.

The following lemma is the main result of this section.

Lemma 5.7. Suppose $L$ is a finite atomistic lattice that admits an equaclosure operator. Then right and left slaloms in L have different parities.

Proof. Let $a, b \in A(L), a \sim b$ and suppose that there are two exact slaloms with the origin at $(a, b)$, a left and a right one, that are both even or both odd. Then applying Lemmas 5.5 and 5.6 we obtain $a \sqsubseteq b$ and $b \sqsubseteq a$. Hence $a=b$ because $\sqsubseteq$ is a partial order on $A(L)$. This yields a contradiction since no non-trivial descent can start from $(a, a)$. Thus right and left slaloms in $L$ must have different parities. 
6. Proof of the theorem. We are now ready to prove the theorem formulated in the introduction.

Assume $L$ is a finite atomistic lattice.

(i) $\Rightarrow$ (ii). Use Propositions 1.1 and 1.2.

(ii) $\Rightarrow$ (iii). Apply Theorem 2.1, remarks following Proposition 3.5 and Lemmas 4.6 and 5.7.

(iv) $\Rightarrow$ (i). This implication is obvious.

(iii) $\Rightarrow$ (iv). Assume $L \cong S u b(P)$ for some finite semilattice $P$. Define $\varphi: P \rightarrow 2^{P}$ by $\varphi(x)=\{y \in P: y \not \leq x\}$ where $y \leq x$ means $y \circ x=y$. Then $\varphi$ embeds the semilattice $P$ in $\left(2^{P} ; \cup\right)$. Indeed, if $x \neq x^{\prime}$ then either $x \not \leq x^{\prime}$ or $x^{\prime} \not \leq x$ and so either $x \in \varphi\left(x^{\prime}\right) \backslash \varphi(x)$ or $x^{\prime} \in \varphi(x) \backslash \varphi\left(x^{\prime}\right)$ which means that $\varphi(x) \neq \varphi\left(x^{\prime}\right)$. As $x \circ x^{\prime} \leq x, x^{\prime}$, it follows that $y \not \leq x \circ x^{\prime}$ whenever $y \not \leq x$ or $y \not \leq x^{\prime}$. Hence $\varphi(x) \cup \varphi\left(x^{\prime}\right) \subseteq \varphi\left(x \circ x^{\prime}\right)$. But if $y \notin \varphi(x)$ and $y \notin \varphi\left(x^{\prime}\right)$ then $y \leq x$ and $y \leq x^{\prime}$ which implies $y \leq x \circ x^{\prime}$ and, therefore, $y \notin \varphi\left(x \circ x^{\prime}\right)$. So $\varphi\left(x \circ x^{\prime}\right)=\varphi(x) \cup \varphi\left(x^{\prime}\right)$. Notice now that the map $A \mapsto \varphi(A)$, where $A \in S u b(P)$, establishes an isomorphism between the lattice $S u b(P)$ and the principal ideal of $S u b\left(2^{P} ; \cup\right)$ generated by $\varphi(P)$. So, as $L \cong S u b(P)$, in order to show that $L \cong L_{q}(\mathbf{K})$ for some quasivariety $\mathbf{K}$ of rings it suffices to show that $\operatorname{Sub}\left(2^{P} ; \cup\right) \cong L_{q}(\mathbf{M})$ for some quasivariety $\mathbf{M}$ of rings and then to take as $\mathbf{K}$ the subquasivariety of $\mathbf{M}$ that corresponds to $\varphi(P)$.

As $P$ is finite, we may assume that $P=\{1, \ldots, n\}$. Let $p_{1}, \ldots, p_{n}$ be a fixed sequence of pairwise different prime numbers and let $Z_{p_{i}}, i=1, \ldots, n$, denote the ring of integers modulo $p_{i}$ which is treated as an abstract algebra with the unit as a nullary operation. Let $\mathbf{M}$ denote the variety of rings generated by all $Z_{p_{i}}$ 's, where $i=1, \ldots, n$. Define $\psi: S u b\left(2^{P} ; \cup\right) \rightarrow L_{q}(\mathbf{M})$ by $\psi(A)=Q\left(\left\{\prod\left(Z_{p_{i}}: i \in X\right): X \in A\right\}\right)$, where $A$ is a subsemilattice of $\left(2^{P} ; \cup\right)$. We show that $\psi$ is a lattice isomorphism.

1-1: Assume $\psi(A)=\psi(B)$. By symmetry, it suffices to show that $A \subseteq B$. Let $X \in A$. Then $\prod\left(Z_{p_{i}}: i \in X\right)$ is in $\psi(A)$ and hence in $\psi(B)$. This implies that $\prod\left(Z_{p_{i}}: i \in X\right)$ is embeddable, say, via an embedding $f$, into the direct product of some algebras (= rings) of the form $\prod\left(Z_{p_{i}}: i \in Y_{j}\right)$ where $j \in J$ and $Y_{j} \in B$. Consider $\pi_{j} \circ f: \prod\left(Z_{p_{i}}: i \in X\right) \rightarrow \prod\left(Z_{p_{i}}: i \in Y_{j}\right)$ where $\pi_{j}$ is the projection of the direct product of $\prod\left(Z_{p_{i}}: i \in Y_{j}\right), j \in J$, onto $\prod\left(Z_{p_{i}}: i \in Y_{j}\right)$. As the latter has no proper subalgebras, we have $\prod\left(Z_{p_{i}}: i \in X\right) / \operatorname{Ker} \pi_{j} \circ f \cong \prod\left(Z_{p_{i}}: i \in Y_{j}\right)$. On the other hand, as each congruence on $\prod\left(Z_{p_{i}}: i \in X\right)$ is a product of congruences on $Z_{p_{i}}$ 's, where $i \in X$, and each $Z_{p_{i}}$ is a simple ring, it follows that $Y_{j} \subseteq X$. But $\bigwedge\left(\operatorname{Ker} \pi_{j} \circ f: j \in J\right)=i d_{\Pi\left(Z_{p_{i}}: i \in X\right)}$. So $\bigcup\left(Y_{j}: j \in J\right)=X$. Hence $X \in B$ and thus $A \subseteq B$.

On to: Let $\mathbf{N} \in L_{q}(\mathbf{M})$, and let $A$ denote the family of subsets $X$ of $P$ such that $\prod\left(Z_{p_{i}}: i \in X\right) \in \mathbf{N}$. As, for $X, Y \in A, \prod\left(Z_{p_{i}}: i \in X \cup Y\right)$ is isomorphic to a subalgebra of $\prod\left(Z_{p_{i}}: i \in X\right) \times \prod\left(Z_{p_{i}}: i \in Y\right)$, it follows 
that $A$ is a subsemilattice of $\left(2^{P} ; \cup\right)$. To show that $\psi(A)=\mathbf{N}$ we need to show that $\mathbf{N}$ is generated by all algebras of the form $\prod\left(Z_{p_{i}}: i \in X\right)$, where $X \in A$. But this is almost immediate since each quasivariety contained in $\mathbf{M}$ is generated by its members that are of the form $\prod\left(Z_{p_{i}}: i \in X\right)$, where $X \subseteq P$. To see this notice that $\mathbf{M}$ is a directly representable variety and its directly indecomposable members are $Z_{p_{i}}$, where $i=1, \ldots, n$.

Meets: As $\psi$ preserves $\leq$, we have $\psi(A B) \subseteq \psi(A) \psi(B)$. So let $R \in$ $\psi(A) \psi(B)$. In view of the previous remarks we may assume that $R$ is of the form $\prod\left(Z_{p_{i}}: i \in X\right)$ where $X \subseteq P$. Then $\prod\left(Z_{p_{i}}: i \in X\right)$ belongs to $\psi(A)$ and to $\psi(B)$. So arguing as in the proof that $\psi$ is one-to-one we find that $X \in A B$. Hence $R \in \psi(A B)$. Thus $\psi(A B)=\psi(A) \psi(B)$.

J o ins: Evidently, $\psi(A)+\psi(B) \subseteq \psi(A+B)$ since $A, B \leq A+B$. As $\psi(A+B)$ is generated by all algebras of the form $\prod\left(Z_{p_{i}}: i \in X\right)$, where $X \in A+B$, to prove the inverse inclusion it suffices to show that, for each $X \in A+B$, the algebra $\prod\left(Z_{p_{i}}: i \in X\right)$ belongs to $\psi(A)+\psi(B)$. But this is obvious since $X=Y \cup Z$ for some $Y \in A$ and $Z \in B$ and since the algebra $\prod\left(Z_{p_{i}}: i \in Y \cup Z\right)$ is isomorphic to a subalgebra of $\prod\left(Z_{p_{i}}: i \in Y\right) \times \prod\left(Z_{p_{i}}: i \in Z\right)$.

Thus $\psi$ is a lattice isomorphism.

7. Two questions. Recall that (see McKenzie [18]) a finite lattice $L$ is said to be lower bounded if $L$ is a homomorphic image of a free lattice by a lattice homomorphism $\phi$ satisfying: for each $a$ in $L$, the set $\phi^{-1}(\{a\})$ has a least element. For some equivalents of this notion see Day [6], Gaskill, Grätzer and Platt [9], and Jónsson and Nation [15]. From our theorem it easily follows that if $L$ is a finite atomistic Q-lattice then $L$ is lower bounded. We wonder whether this remains valid without the assumption that $L$ is atomistic. In other words, we ask the following

Question 1. Is every finite Q-lattice lower bounded?

The first author has recently proved that a finite lattice is lower bounded if and only if it is isomorphic to a sublattice of $S u b(P)$ for some finite semilattice $P$. So if the question has an affirmative answer then we can localize the finite Q-lattices as the sublattices of the lattices of finite semilattices. This in our opinion would give an essential step towards finding a complete solution of Mal'cev problem for finite Q-lattices. We also want to mention that the question was verified in [3] for finite Q-lattices that come from locally finite quasivarieties. In [3], it was shown that $L_{q}(\mathbf{K})$ is lower bounded provided that $\mathbf{K}$ is a locally finite quasivariety and $L_{q}(\mathbf{K})$ is finite. However, we do not know whether or not the lattice of Figure 4, called by us the leaf, is a Q-lattice. Notice that it is not lower bounded. 


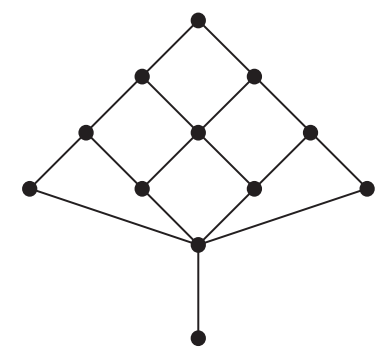

Fig. 4

The above remarks and Propositions 1.1 and 1.2 also suggest the following

Question 2. Given a finite lattice $L$ that is lower bounded, biatomic and admits an equa-closure operator. Is $L$ a Q-lattice?

The authors would like to thank Professors Ralph Freese, Keith Kearnes and J. B. Nation for their correspondence, and for providing us with their paper Congruence lattices of congruence semidistributive algebras, preprint (1991), where the above Question 1 is also raised and verified within Qlattices of locally finite quasivarieties. The authors would also like to thank the referee for valuable suggestions improving the presentation of the paper.

\section{References}

[1] K. V. Adaricheva, A characterization of finite lattices of subsemilattices, Algebra i Logika 30 (1991), 385-404 (in Russian).

[2] K. V. Adaricheva and V. A. Gorbunov, Equaclosure operator and forbidden semidistributive lattices, Sibirsk. Mat. Zh. 30 (1989), 7-25 (in Russian).

[3] K. V. Adaricheva, W. Dziobiak and V. A. Gorbunov, The lattices of quasivarieties of locally finite quasivarieties, preprint.

[4] M. K. Bennett, Biatomic lattices, Algebra Universalis 24 (1987), 60-73.

[5] G. Birkhoff and M. K. Bennett, The convexity lattice of a poset, Order 2 (1985), 223-242.

[6] A. Day, Characterization of finite lattices that are bounded-homomorphic image of sublattices of free lattices, Canad. J. Math. 31 (1979), 69-78.

[7] W. Dziobiak, On atoms in the lattice of quasivarieties, Algebra Universalis 24 (1987), 31-35.

[8] R. Freese and J. B. Nation, Congruence lattices of semilattices, Pacific J. Math. 44 (1973), 51-58.

[9] H. Gaskill, G. Grätzer and C. R. Platt, Sharply transferable lattices, Canad. J. Math. 27 (1975), 1246-1262.

[10] V. A. Gorbunov, Lattices of quasivarieties, Algebra i Logika 15 (1976), 436-457 (in Russian).

[11] V. A. Gorbunov and V. I. Tumanov, A class of lattices of quasivarieties, ibid. 19 (1980), 59-80 (in Russian). 
[12] V. A. Gorbunov and V. I. Tumanov, The structure of the lattices of quasivarieties, in: Trudy Inst. Mat. (Novosibirsk) 2, Nauka Sibirsk. Otdel., Novosibirsk 1982, 12-44 (in Russian).

[13] G. Grätzer, General Lattice Theory, Birkhäuser, Basel 1979.

[14] G. Grätzer and H. Lakser, A note on the implicational class generated by a class of structures, Canad. Math. Bull. 16 (1973), 603-605.

[15] B. Jónsson and J. B. Nation, A report on sublattices of a free lattice, in: Contributions to Universal Algebra, Szeged 1975, Colloq. Math. Soc. János Bolyai 17, 223-257.

[16] A. I. Mal'cev, On certain frontier questions in algebra and mathematical logic, in: Proc. Int. Congr. Mathematicians, Moscow 1966, Mir, 1968, 217-231 (in Russian).

[17] —, Algebraic Systems, Springer, 1973.

[18] R. McKenzie, Equational bases and nonmodular lattice varieties, Trans. Amer. Math. Soc. 174 (1972), 1-43.

[19] R. McKenzie, G. McNulty and W. Taylor, Algebras, Lattices, Varieties, Wadsworth and Brooks/Cole, Monterey 1987.

[20] V. I. Tumanov, Finite distributive lattices of quasivarieties, Algebra i Logika 22 (1983), 168-181 (in Russian).

INSTITUTE OF MATHEMATICS

RUSSIAN ACADEMY OF SCIENCES

UNIVERSITETSKII PROSP. 4

630090 NOVOSIBIRSK, RUSSIA
INSTITUTE OF MATHEMATICS

NICHOLAS COPERNICUS UNIVERSITY

CHOPINA $12 / 18$

87-100 TORUŃ, POLAND

Received 20 May 1991;

in revised form 3 March 1992 